\title{
Spin Polarization Curve of Fractional Quantum Hall States with Filling Factor Smaller than 2
}

\author{
Shosuke Sasaki \\ Center for Quantum Science and Technology under Extreme Conditions (KYOKUGEN), Osaka University, \\ 1-3 Machikaneyama, Toyonaka, Osaka 560-8531, Japan \\ Correspondence should be addressed to Shosuke Sasaki; sasaki@ns.sist.ac.jp
}

Received 30 June 2013; Accepted 12 August 2013

Academic Editors: H. Hibino and A. N. Kocharian

Copyright (C) 2013 Shosuke Sasaki. This is an open access article distributed under the Creative Commons Attribution License, which permits unrestricted use, distribution, and reproduction in any medium, provided the original work is properly cited.

Kukushkin et al. have measured the electron spin polarization versus magnetic field in the fractional quantum Hall states. The polarization curves show wide plateaus and small shoulders. The $2 \mathrm{D}$ electron system is described by the total Hamiltonian $\left(H_{D}+H_{I}\right)$. Therein, $H_{D}$ is the sum of the Landau energies and classical Coulomb energies. $H_{I}$ is the residual interaction yielding Coulomb transitions. It is proven for any filling factor that the most uniform electron configuration in the Landau states is only one. The configuration has the minimum energy of $H_{D}$. When the magnetic field is weak, some electrons have up-spins and the others downspins. Then, there are many spin arrangements. These spin arrangements give the degenerate ground states of $H_{D}$. We consider the partial Hamiltonian only between the ground states. The partial Hamiltonian yields the Peierls instability and is diagonalized exactly. The sum of the classical Coulomb and spin exchange energies has minimum for an interval modulation between Landau orbitals. Using the solution with the minimum energy, the spin polarization is calculated which reproduces the wide plateaus and small shoulders. The theoretical result is in good agreement with the experimental data.

\section{Introduction}

In this paper, we examine the electron spin polarization in the FQH states with the filling factor $v<2$. Before the examination, we see here the investigations on the FQHE briefly.

The fractional quantum Hall effect was discovered by Pan et al. $[1,2]$. The quasi particle with a fractional charge and its wave function were introduced by Laughlin using the variational method $[3,4]$. Many physicists developed it [5-7]. Jain proposed the composite fermion theory [8, 9]. Thereafter, the FQH states with the nonstandard filling factors have been investigated by employing various methods as in the references [10-14]. These theories assume the various types of the quasi particles and their mixing. On the other hand, Tao and Thouless $[15,16]$ examined the case that the lowest Landau levels are partially filled with electrons. Their method is very important to investigate the $\mathrm{FQH}$ states. We have developed the Tao-Thouless theory and have found the most uniform configuration of electrons. It has been proven that the configuration is unique for any filling factor [17]. The configuration minimizes the expectation value of the total Hamiltonian.

The Coulomb transitions conserve the $x$ component of the total momentum where the $x$-direction indicates the current direction. The conservation law produces energy gaps for the specific filling factors. For the other filling factors, we have found the gapless structure and peak structure [17-23]. The theory can well explain the behaviors of the FQHE at $v<2$ without any quasi particles.

On the other hand, the electrons in FQH states with $v>2$ occupy the higher Landau levels. Therein, many experimental and theoretical investigations [24-43] are carried out and various interesting phenomena are discovered. Also, the electron spin polarization is investigated for the $\mathrm{FQH}$ states with $v>2$ by the papers [44-49].

Although the function form of the spin-polarization versus magnetic field is very important for investigating the FQHE, there is no theoretical calculation of the function shape quantitatively. In this paper, we calculate the spinpolarization versus magnetic field at $\nu<2$ by developing the previous method in the references [17-23]. 

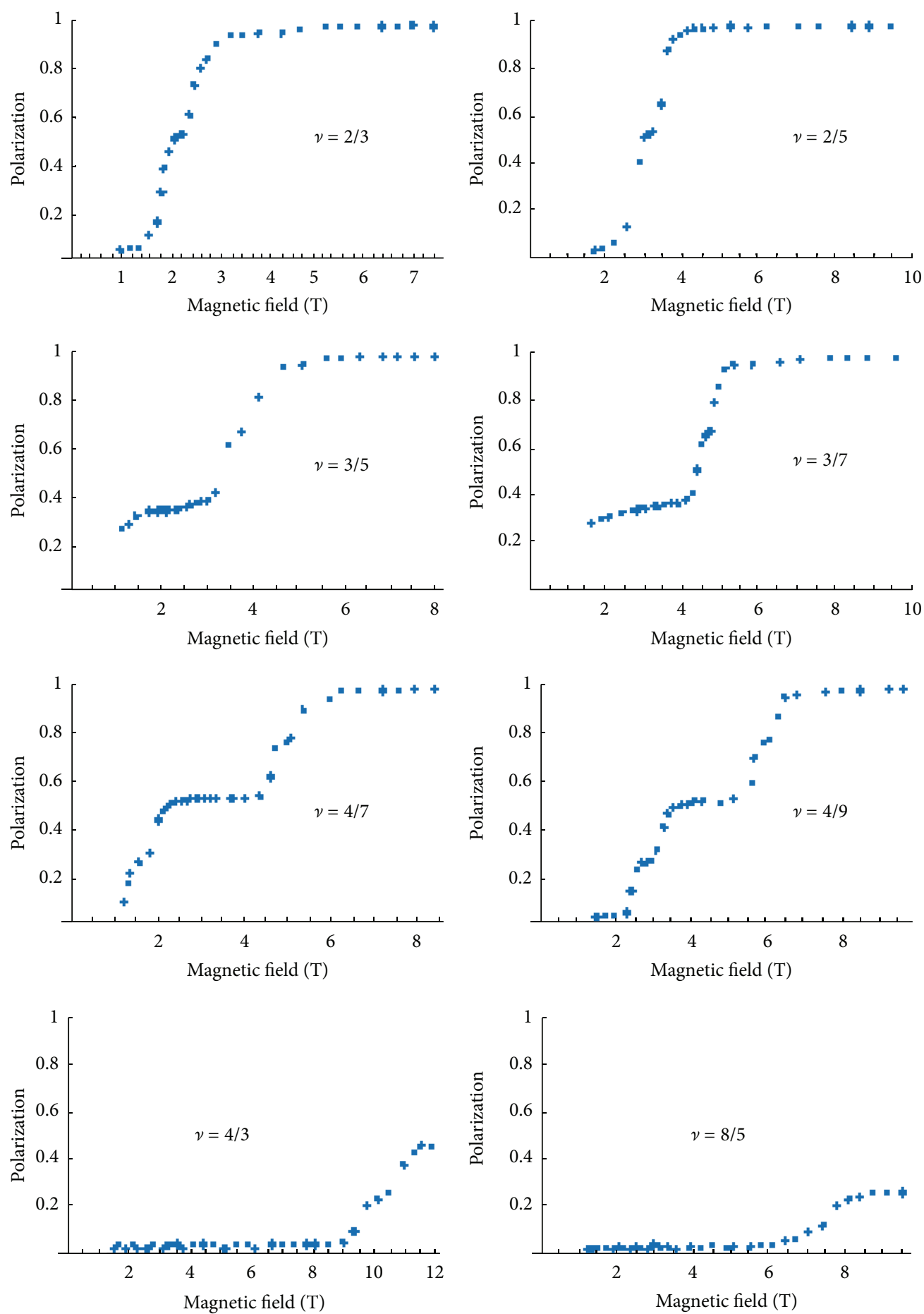

FIGURE 1: Experimental data of the polarization for the FQH states [50].

Kukushkin et al. have measured the electron spinpolarization versus magnetic field [50]. They clarified the function forms at twelve filling factors in $v<2$. Although their experiments are rather old data, the obtained function forms give us the important knowledge.

Their results are shown in Figure 1. Hereafter, we describe the electron spin polarization by the symbol $\gamma_{e}$. Then, $\gamma_{e}=1$ means a fully polarized state. The experimental polarization curves have the following properties.
(1) The wide plateau appears at $\gamma_{e}=0$ for $\nu=2 / 3$ and $2 / 5$.

The wide plateau appears at $\gamma_{e}=1 / 3$ for $\nu=3 / 5$ and $3 / 7$.

The two wide plateaus appear at $\gamma_{e}=0$ and $1 / 2$ for $v=4 / 7$ and $4 / 9$.

(2) The small shoulder appears at $\gamma_{e}=1 / 2$ for $\nu=2 / 3$ and $2 / 5$. 
The small shoulder appears at $\gamma_{e}=2 / 3$ for $v=3 / 5$ and $3 / 7$.

The two small shoulder appear at $\gamma_{e}=1 / 4$ and $3 / 4$ for $\nu=4 / 7$ and $4 / 9$.

As shown in Figure 1, the $v=2 / 3$ polarization curve resembles the $v=2 / 5$ curve. The $v=3 / 5$ polarization curve resembles the $v=3 / 7$ curve. Also, the $v=4 / 7$ curve resembles the $v=4 / 9$ curve. The characters mentioned above indicate that the shape of the spin polarization curves depends mainly upon the numerator of the filling factor. The numerator means the electron number per unit configuration (see [17]). Therefore the polarization belongs to electrons (not holes). In this paper we clarify the origin of the polarization curve.

We shortly describe the fundamental properties of the quasi-two-dimensional electron system below. We illustrate a quantum Hall device where the directions of the axes $x, y$, and $z$ are taken, as in Figure 2. Then, the vector potential, A, has the components:

$$
\mathbf{A}=(-y B, 0,0),
$$

where $B$ is the strength of the magnetic field.

The Hamiltonian, $H_{0}$, of a single electron in the absence of the Coulomb interaction between electrons is given by

$$
H_{0}=\frac{(\mathbf{p}+e \mathbf{A})^{2}}{2 m^{*}}+U(y)+W(z),
$$

where $U(y)$ and $W(z)$ indicate the potentials confining electrons to an ultrathin conducting layer in Figure 2. Therein $m^{*}$ is an effective mass of electron, and $\mathbf{p}=\left(p_{x}, p_{y}, p_{z}\right)$ is the electron momentum. The effective mass $m^{*}$ differs from material to material and the value in GaAs is about 0.067 times the free electron mass. The eigenvalue problem of this Hamiltonian is solved and the single electron wave function $\psi_{L, J}$ is expressed as follows:

$$
\begin{aligned}
\psi_{L, J}(x, y, z)= & \sqrt{\frac{1}{\ell}} \exp \left(\frac{i p x}{\hbar}\right) u_{L} H_{L}\left(\sqrt{\frac{m^{*} \omega}{\hbar}}\left(y-\alpha_{J}\right)\right) \\
& \times \exp \left(-\frac{m^{*} \omega}{2 \hbar}\left(y-\alpha_{J}\right)^{2}\right) \phi(z),
\end{aligned}
$$

where $\alpha_{J}$ is given by

$$
\alpha_{J}=\frac{p}{(e B)}=\left[\frac{2 \pi \hbar}{(\ell e B)}\right] J, \quad \ell: \text { length of } 2 \mathrm{D} \text { electron system. }
$$

Therein, $\phi(z)$ is the wave function of the ground state along the $z$-direction, $H_{L}$ is the Hermite polynomial of $L$ th degree and $u_{L}$ is the normalization constant. We call $L$ the Landau level number. Also, the eigenenergy is given by

$$
E_{L, J}=\lambda+U\left(\alpha_{J}\right)+\hbar \omega\left(L+\frac{1}{2}\right), \quad(L=0,1,2,3, \ldots),
$$

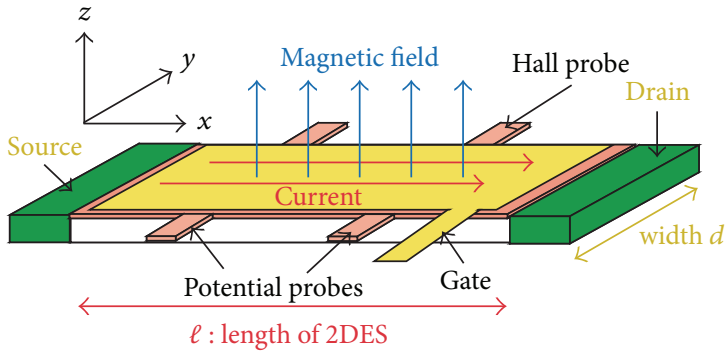

FIGURE 2: Quantum Hall device.

where $\lambda$ is the ground state energy along the $z$-direction $U\left(\alpha_{J}\right)$ is the potential energy in the $y$-direction.

When there are many electrons, the total Hamiltonian is given by

$$
\begin{aligned}
H_{T}= & \sum_{i=1}^{N} H_{0, i} \\
& +\sum_{i=1}^{N-1} \sum_{j>i}^{N} \frac{e^{2}}{4 \pi \varepsilon \sqrt{\left(x_{i}-x_{j}\right)^{2}+\left(y_{i}-y_{j}\right)^{2}+\left(z_{i}-z_{j}\right)^{2}}},
\end{aligned}
$$

where $N$ is the total number of electrons and $H_{0, i}$ is the single particle Hamiltonian of the $i$ th electron without the Coulomb interaction as

$$
H_{0, i}=\frac{\left(\mathbf{p}_{i}+e \mathbf{A}\right)^{2}}{2 m^{*}}+U\left(y_{i}\right)+W\left(z_{i}\right) .
$$

The many-electron state is characterized by a set of Landau level numbers $L_{1}, L_{2}, \ldots, L_{N}$ and a set of momenta $p_{1}, p_{2}, \ldots, p_{N}$. The complete set is composed of the Slater determinant as

$$
\begin{aligned}
& \Psi\left(L_{1}, \ldots, L_{N} ; p_{1}, \ldots, p_{N}\right) \\
& =\frac{1}{\sqrt{N !}}\left|\begin{array}{ccc}
\psi_{L_{1}, p_{1}}\left(x_{1}, y_{1}, z_{1}\right) & \cdots & \psi_{L_{1}, p_{1}}\left(x_{N}, y_{N}, z_{N}\right) \\
\vdots & & \vdots \\
\psi_{L_{N}, p_{N}}\left(x_{1}, y_{1}, z_{1}\right) & \cdots & \psi_{L_{N}, p_{N}}\left(x_{N}, y_{N}, z_{N}\right)
\end{array}\right| .
\end{aligned}
$$

This state is the eigenstate of $\sum_{i=1}^{N} H_{0, i}$. The expectation value of the total Hamiltonian is denoted as $W\left(L_{1}, \ldots, L_{N} ; p_{1}, \ldots, p_{N}\right)$ which is given by

$$
\begin{aligned}
& W\left(L_{1}, \ldots, L_{N} ; p_{1}, \ldots, p_{N}\right) \\
& \quad=\sum_{i=1}^{N} E_{L_{i}}\left(p_{i}\right)+C\left(L_{1}, \ldots, L_{N} ; p_{1}, \ldots, p_{N}\right),
\end{aligned}
$$

where $C$ is the expectation value of the Coulomb interaction defined by

$$
\begin{aligned}
& C\left(L_{1}, \ldots, L_{N} ; p_{1}, \ldots, p_{N}\right) \\
& =\int \ldots \int \Psi\left(L_{1}, \ldots, L_{N} ; p_{1}, \ldots, p_{N}\right)^{*}
\end{aligned}
$$




$$
\begin{aligned}
& \times \sum_{i=1}^{N-1} \sum_{j>i}^{N} \frac{e^{2}}{4 \pi \varepsilon \sqrt{\left(x_{i}-x_{j}\right)^{2}+\left(y_{i}-y_{j}\right)^{2}+\left(z_{i}-z_{j}\right)^{2}}} \\
& \times \Psi\left(L_{1}, \ldots, L_{N} ; p_{1}, \ldots, p_{N}\right) \mathrm{d} x_{1} \mathrm{~d} y_{1} \mathrm{~d} z_{1}, \ldots, \mathrm{d} x_{N} \mathrm{~d} y_{N} \mathrm{~d} z_{N}
\end{aligned}
$$

Hereafter, we call $C\left(L_{1}, \ldots, L_{N} ; p_{1}, \ldots, p_{N}\right)$ "classical Coulomb energy." We divide the total Hamiltonian $H_{T}$ into two parts $H_{D}$, and $H_{I}$, as follows:

$$
\begin{aligned}
H_{D}=\sum_{L_{1}, \ldots, L_{N}} \sum_{p_{1}, \ldots, p_{N}} \mid & \left.\Psi\left(L_{1}, \ldots, L_{N} ; p_{1}, \ldots, p_{N}\right)\right\rangle \\
& \times W\left(L_{1}, \ldots, L_{N} ; p_{1}, \ldots, p_{N}\right) \\
& \times\left\langle\Psi\left(L_{1}, \ldots, L_{N} ; p_{1}, \ldots, p_{N}\right)\right| \\
H_{I} & =H_{T}-H_{D},
\end{aligned}
$$

where $H_{\mathrm{I}}$ is constructed only by the off-diagonal elements and depends upon only the relative coordinate. Therefore, the total momentum of the $x$-direction conserves in this system. That is to say, the sum of the initial momenta $p_{i}$ and $p_{j}$ is equal to the sum of the final momenta $p_{i}^{\prime}$ and $p_{j}^{\prime}$ via Coulomb transition as follows:

$$
p_{i}^{\prime}+p_{j}^{\prime}=p_{i}+p_{j}
$$

At a filling factor smaller than 1 , the ground state of $H_{D}$ satisfies the following properties.

(1) $N$ electrons exist in the lowest Landau levels with $L_{1}=L_{2}=\cdots=L_{N}=0$.

(2) The electrons most uniformly occupy the lowest Landau levels. Then, the classical Coulomb energy takes the lowest one. The electron momenta $p_{1}, p_{2}, \ldots, p_{N}$ are related to each centre position as in (4).

For any filling factor, we can find only one electron configuration in Landau states which is the most uniform and has the minimum energy of $H_{D}$. The proof is done in [17]. When the magnetic field is weak, there are many spin arrangements for a given configuration. These spin arrangements construct the degenerate ground states of $H_{D}$. The interaction Hamiltonian $H_{I}$ yields the quantum transitions among the ground states. We examine the interaction in the next section.

\section{Coulomb Interaction between Up- and Down-Spin States (Equivalency between Coulomb Transition and Spin Exchange Interaction)}

The degenerate ground states have the same momentum set corresponding to the most uniform electron configuration. The Hamiltonian $H_{I}$ given by (11) acts between two electrons.
We indicate the spin states by $\uparrow$ and $\downarrow$ for up- and downspins, respectively. Then, all the initial spin states with the momentum pair $p_{1}, p_{2}$ are described as

$$
\begin{array}{ll}
\left|p_{1} \uparrow, p_{2} \uparrow\right\rangle, & \left|p_{1} \uparrow, p_{2} \downarrow\right\rangle, \\
\left|p_{1} \downarrow, p_{2} \uparrow\right\rangle, & \left|p_{1} \downarrow, p_{2} \downarrow\right\rangle .
\end{array}
$$

When these states transfer via $H_{I}$, their final states are described as follows:

$$
\begin{array}{ll}
\left|p_{1}^{\prime} \uparrow, p_{2}^{\prime} \uparrow\right\rangle, & \left|p_{1}^{\prime} \uparrow, p_{2}^{\prime} \downarrow\right\rangle, \\
\left|p_{1}^{\prime} \downarrow, p_{2}^{\prime} \uparrow\right\rangle, & \left|p_{1}^{\prime} \downarrow, p_{2}^{\prime} \downarrow\right\rangle,
\end{array}
$$

where $p_{1}^{\prime}$ and $p_{2}^{\prime}$ indicate the final momenta via the Coulomb interaction. We consider the transitions only between the degenerate ground states. Therefore, the final momentum set should have the minimum energy of $H_{D}$. Accordingly, the final momentum set is equivalent to the initial momentum set because of the uniqueness of the electron configuration for the ground state of $H_{D}$ :

$$
p_{1}^{\prime}=p_{2}, \quad p_{2}^{\prime}=p_{1} .
$$

The case of $\left(p_{1}^{\prime}=p_{1}, p_{2}^{\prime}=p_{2}\right)$ is removed because the diagonal matrix elements of $H_{\mathrm{I}}$ are zero. Applying (15), the final state $\left|p_{1}^{\prime} \uparrow, p_{2}^{\prime} \uparrow\right\rangle$ becomes $\left|p_{2} \uparrow, p_{1} \uparrow\right\rangle$ which is the same as its initial state. Also $\left|p_{1}^{\prime} \downarrow, p_{2}^{\prime} \downarrow\right\rangle$ becomes $\left|p_{2} \downarrow, p_{1} \downarrow\right\rangle$. In the two cases, the final state is identical to the initial state, and therefore the matrix elements of $H_{I}$ are zero. Accordingly, nonzero matrix elements are

$$
\begin{aligned}
& \left\langle p_{2} \uparrow, p_{1} \downarrow\left|H_{\mathrm{I}}\right| p_{1} \uparrow, p_{2} \downarrow\right\rangle, \\
& \left\langle p_{2} \downarrow, p_{1} \uparrow\left|H_{\mathrm{I}}\right| p_{1} \downarrow, p_{2} \uparrow\right\rangle,
\end{aligned}
$$

where

$$
\left\langle p_{2} \downarrow, p_{1} \uparrow\left|H_{\mathrm{I}}\right| p_{1} \downarrow, p_{2} \uparrow\right\rangle=\left\langle p_{2} \uparrow, p_{1} \downarrow\left|H_{\mathrm{I}}\right| p_{1} \uparrow, p_{2} \downarrow\right\rangle .
$$

We examine the following three cases.

Case 1. Consider $p_{1}-p_{2}= \pm 2 \pi \hbar / \ell$

$$
\xi=\left\langle p_{2} \uparrow, p_{1} \downarrow\left|H_{\mathrm{I}}\right| p_{1} \uparrow, p_{2} \downarrow\right\rangle .
$$

Case 2. Consider $p_{3}-p_{4}= \pm 4 \pi \hbar / \ell$

$$
\eta=\left\langle p_{4} \uparrow, p_{3} \downarrow\left|H_{\mathrm{I}}\right| p_{3} \uparrow, p_{4} \downarrow\right\rangle .
$$

Case 3. Consider $p_{5}-p_{6}= \pm 6 \pi \hbar / \ell$

$$
\varsigma=\left\langle p_{6} \uparrow, p_{5} \downarrow\left|H_{\mathrm{I}}\right| p_{5} \uparrow, p_{6} \downarrow\right\rangle .
$$

The Coulomb transition in Case 1 is shown in Figure 3. The open circle indicates the up-spin state and the filled one the down-spin state. We describe the momenta after the transition by the symbols $p_{1}^{\prime}$ and $p_{2}^{\prime}$ which are given by

$$
p_{1}^{\prime}=p_{1}+\frac{2 \pi \hbar}{\ell}=p_{2}, \quad p_{2}^{\prime}=p_{2}-\frac{2 \pi \hbar}{\ell}=p_{1} .
$$




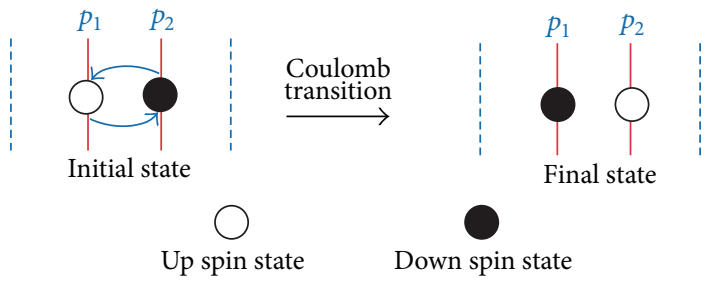

Figure 3: Equivalence of a specific Coulomb transition and spin exchange interaction for Case 1.

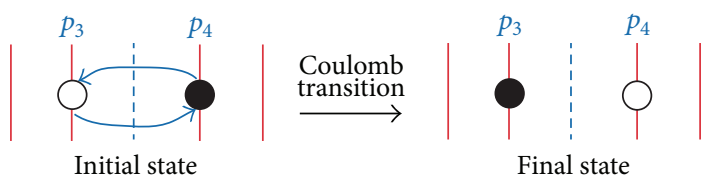

FIgURE 4: Equivalence of a specific Coulomb transition and spin exchange interaction for Case 2.

This Coulomb transition is equivalent to the following process: The spin at site 1 flips from up to down and the spin at site 2 flips from down to up simultaneously without changing the momenta. Thus, the Coulomb transition of Case 1 is equivalent to a spin exchange process which is described by the interaction $\xi \sigma_{1}^{-} \sigma_{2}^{+}$. Therein, $\sigma^{+}$is the spin transformation operator from down- to up-spin state and $\sigma^{-}$is the adjoint operator of $\sigma^{+}$. There is another Coulomb transition given by (16b) which is equivalent to $\xi \sigma_{1}^{+} \sigma_{2}^{-}$. Accordingly, the Coulomb transition between the two electrons at sites 1 and 2 is expressed as

$$
\xi\left(\sigma_{1}^{-} \sigma_{2}^{+}+\sigma_{1}^{+} \sigma_{2}^{-}\right)
$$

where $\xi$ was already defined by (18a). In this Coulomb transition, the classical Coulomb energy in the initial state is exactly equal to the one in the final state.

The Coulomb transition of Case 2 is illustrated in Figure 4 .

The momenta after the transition are described by the symbols $p_{3}^{\prime}, p_{4}^{\prime}$, the values of which are given by

$$
p_{3}^{\prime}=p_{3}+\frac{2 \times 2 \pi \hbar}{\ell}=p_{4}, \quad p_{4}^{\prime}=p_{4}-\frac{2 \times 2 \pi \hbar}{\ell}=p_{3} .
$$

The Coulomb interaction of Case 2 is equivalent to the following spin exchange interaction between an electron pair placed in second nearest-neighboring orbital pair:

$$
\eta\left(\sigma_{3}^{-} \sigma_{4}^{+}+\sigma_{3}^{+} \sigma_{4}^{-}\right)
$$

where $\eta$ is the coupling constant defined by (18b).

The Coulomb transition of Case 3 is illustrated in Figure 5. The momenta after the transition are described by the symbols $p_{5}^{\prime}, p_{6}^{\prime}$ :

$$
p_{5}^{\prime}=p_{5}+\frac{3 \times 2 \pi \hbar}{\ell}=p_{6}, \quad p_{6}^{\prime}=p_{6}-\frac{3 \times 2 \pi \hbar}{\ell}=p_{5} .
$$

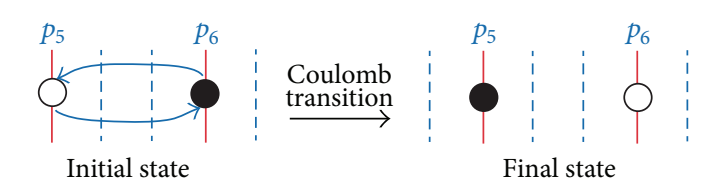

FIgURE 5: Equivalence of a specific Coulomb transition and spin exchange interaction for Case 3.

Figure 5 shows that the Coulomb interaction of Case 3 is equivalent to the following spin exchange interaction between an electron pair placed in third nearest-neighboring orbital pair:

$$
\varsigma\left(\sigma_{5}^{-} \sigma_{6}^{+}+\sigma_{5}^{+} \sigma_{6}^{-}\right)
$$

We show the most uniform configuration of electrons for the two cases of $v=2 / 3$ and $v=2 / 5$ in Figures 6(a) and 6(b), respectively, where the spin-states are numbered sequentially from left to right, as indicated by the green color.

In the $v=2 / 3$ state, the nearest electron pairs have the coupling constant $\xi$ and the second nearest electron pairs have the coupling constant $\eta$. At $\nu=2 / 5$, the nearest electron pair is placed in the second neighboring orbital pair, and so the coupling constant is $\eta$. The second nearest electron pair is placed in the third neighboring orbital pair and so the coupling constant is $\zeta$ as shown in Figure 6(b). Thus, it is noteworthy that the site number (namely, electron number) is different from the orbital number.

In the third or further nearest electron pair, another electron is inserted as in Figures 6(a) and 6(b). Therefore the interaction between the third nearest electrons is quite weak due to the screening effect of the interposing electron, and so may be neglected.

At $v=2 / 3$ the most effective interaction is obtained as follows:

$$
\begin{aligned}
H_{\text {effective }}=\sum_{j=1,2,3, \ldots}[ & \xi\left(\sigma_{2 j-1}^{+} \sigma_{2 j}^{-}+\sigma_{2 j-1}^{-} \sigma_{2 j}^{+}\right) \\
& \left.+\eta\left(\sigma_{2 j}^{+} \sigma_{2 j+1}^{-}+\sigma_{2 j}^{-} \sigma_{2 j+1}^{+}\right)\right],
\end{aligned}
$$

where the operator $\sigma_{2 j-1}^{+}$indicates the transformation from a down- to up-spin state of the electron at the $(2 j-1)$ th site. This Hamiltonian, (25), yields the quantum transition between the degenerate ground states. When the external magnetic field is applied in the $z$-direction, the Hamiltonian becomes

$$
\begin{aligned}
H= & \sum_{j=1,2,3, \ldots}\left[\xi\left(\sigma_{2 j-1}^{+} \sigma_{2 j}^{-}+\sigma_{2 j-1}^{-} \sigma_{2 j}^{+}\right)\right. \\
& \left.+\eta\left(\sigma_{2 j}^{+} \sigma_{2 j+1}^{-}+\sigma_{2 j}^{-} \sigma_{2 j+1}^{+}\right)\right] \\
& +\sum_{i=1,2,3, \ldots} \mu_{B} g^{*} B\left(\frac{1}{2}\right) \sigma_{i}^{z},
\end{aligned}
$$

where $g^{*}$ is the effective $g$-factor, $B$ is the magnetic field, $(1 / 2) \sigma^{z}$ is the electron spin operator in the $z$-direction, 


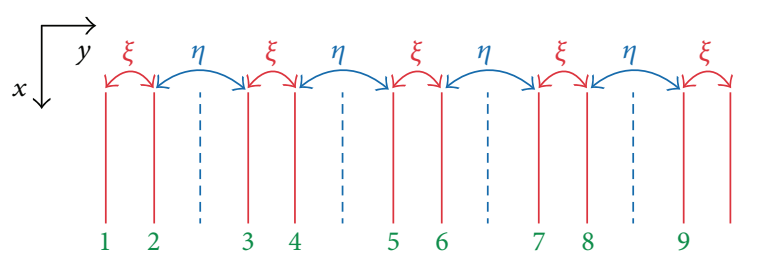

(a)

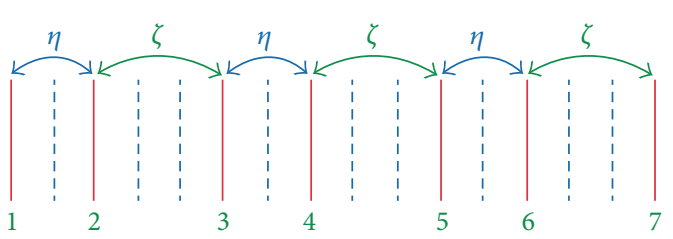

(b)

FIgURE 6: (a) Coulomb transitions for first and second nearest pairs at $v=2 / 3$. (b) Coulomb transitions for the case of $v=2 / 5$.

and $\mu_{B}$ is the Bohr magneton. The matrices (the Pauli spin matrices) are explicitly indicated below:

$$
\sigma^{+}=\left(\begin{array}{ll}
0 & 1 \\
0 & 0
\end{array}\right), \quad \sigma^{-}=\left(\begin{array}{ll}
0 & 0 \\
1 & 0
\end{array}\right), \quad \sigma^{z}=\left(\begin{array}{cc}
1 & 0 \\
0 & -1
\end{array}\right) .
$$

We can obtain the Hamiltonian $H$ for other filling factors. The Hamiltonian for $v=3 / 5$ and $v=4 / 7$ is given, respectively by;

$$
\begin{aligned}
& H=\sum_{j=1,2,3, \ldots}\left[\xi\left(\sigma_{3 j-2}^{+} \sigma_{3 j-1}^{-}+\sigma_{3 j-2}^{-} \sigma_{3 j-1}^{+}\right)\right. \\
&+ \eta\left(\sigma_{3 j-1}^{+} \sigma_{3 j}^{-}+\sigma_{3 j-1}^{-} \sigma_{3 j}^{+}\right) \\
&\left.+\eta\left(\sigma_{3 j}^{+} \sigma_{3 j+1}^{-}+\sigma_{3 j}^{-} \sigma_{3 j+1}^{+}\right)\right] \\
&+\sum_{i=1,2,3, \ldots} \mu_{B} g^{*} B\left(\frac{1}{2}\right) \sigma_{i}^{z}, \quad \text { for } v=\frac{3}{5}, \\
& H=\sum_{j=1,2,3, \ldots}\left[\xi\left(\sigma_{4 j-3}^{+} \sigma_{4 j-2}^{-}+\sigma_{4 j-3}^{-} \sigma_{4 j-2}^{+}\right)\right. \\
&+\eta\left(\sigma_{4 j-2}^{+} \sigma_{4 j-1}^{-}+\sigma_{4 j-2}^{-} \sigma_{4 j-1}^{+}\right) \\
&+\eta\left(\sigma_{4 j-1}^{+} \sigma_{4 j}^{-}+\sigma_{4 j-1}^{-} \sigma_{4 j}^{+}\right) \\
&\left.+\eta\left(\sigma_{4 j}^{+} \sigma_{4 j+1}^{-}+\sigma_{4 j}^{-} \sigma_{4 j+1}^{+}\right)\right] \\
&+\sum_{i=1,2,3, \ldots} \mu_{B} g^{*} B\left(\frac{1}{2}\right) \sigma_{i}^{z}, \quad \text { for } v=\frac{4}{7} .
\end{aligned}
$$

The three Hamiltonians, (26) and (28), can be exactly diagonalized by using the method of [51].

\section{Isomorphic Mapping from the FQH State to One-Dimensional Fermion State}

We examine the following mapping from a single spin state to a fermion state. The down-spin state $|\downarrow\rangle$ is mapped to the vacuum state $|0\rangle$, and the up-spin state $|\uparrow\rangle$ is mapped to the one fermion state $c^{*}|0\rangle$, where $c^{*}$ is the creation operator

$$
|\uparrow\rangle \longrightarrow c *|0\rangle, \quad|\downarrow\rangle \longrightarrow|0\rangle .
$$

Then the spin operators $\sigma^{+}, \sigma^{-}$, and $\sigma^{z}$ are mapped to the operators of the fermion system as

$$
\sigma^{+} \longrightarrow c^{*}, \sigma^{-} \longrightarrow c, \quad \sigma^{z} \longrightarrow\left(2 c^{*} c-1\right) .
$$

Next, we find the isomorphic mapping from many-spin states to many-fermion states. Two examples of the mapping are written as follows:

$$
\begin{aligned}
& \left|\uparrow_{1}, \uparrow_{2}, \downarrow_{3}, \uparrow_{4}, \downarrow_{5}, \downarrow_{6}, \uparrow_{7}, \uparrow_{8}, \downarrow_{9}, \uparrow_{10}\right\rangle \longleftrightarrow c_{1}^{*} c_{2}^{*} c_{4}^{*} c_{7}^{*} c_{8}^{*} c_{10}^{*}|0\rangle, \\
& \left|\downarrow_{1}, \uparrow_{2}, \uparrow_{3}, \downarrow_{4}, \downarrow_{5}, \uparrow_{6}, \downarrow_{7}, \downarrow_{8}, \downarrow_{9}, \uparrow_{10}\right\rangle \longleftrightarrow c_{2}^{*} c_{3}^{*} c_{6}^{*} c_{10}^{*}|0\rangle .
\end{aligned}
$$

It is noteworthy that the multiplying order of creation operators is the same as the order of the up-spins. The operators $c_{i}$ and $c_{i}^{*}$ satisfy the anticommutation relations as follows:

$$
\begin{gathered}
\left\{c_{i}, c_{j}^{*}\right\}=c_{i} \times c_{j}^{*}+c_{j}^{*} \times c_{i}=\delta_{i, j}, \\
\left\{c_{i}, c_{j}\right\}=c_{i} \times c_{j}+c_{j} \times c_{i}=0, \\
\left\{c_{i}^{*}, c_{j}^{*}\right\}=c_{i}^{*} \times c_{j}^{*}+c_{j}^{*} \times c_{i}^{*}=0 .
\end{gathered}
$$

The products of the spin operators $\sigma_{2 j-1}^{+} \sigma_{2 j}^{-}, \sigma_{2 j-1}^{-} \sigma_{2 j}^{+}, \sigma_{2 j}^{+} \sigma_{2 j+1}^{-}$ and $\sigma_{2 j}^{-} \sigma_{2 j+1}^{+}$are mapped to the products of fermion operators as follows:

$$
\begin{gathered}
\sigma_{2 j-1}^{+} \sigma_{2 j}^{-} \longleftrightarrow c_{2 j-1}^{*} c_{2 j}, \quad \sigma_{2 j-1}^{-} \sigma_{2 j}^{+} \longleftrightarrow-c_{2 j-1} c_{2 j}^{*}, \\
\sigma_{2 j}^{+} \sigma_{2 j+1}^{-} \longleftrightarrow c_{2 j}^{*} c_{2 j+1}, \quad \sigma_{2 j}^{-} \sigma_{2 j+1}^{+} \longleftrightarrow-c_{2 j} c_{2 j+1}^{*}, \\
\sigma_{i}^{z} \longleftrightarrow 2 c_{i}^{*} c_{i}-1 .
\end{gathered}
$$

It has been verified that the mapping ((33a), (33b), (33c)) is isomorphic (see [51]). Accordingly Hamiltonian (26) is equivalent to the following form:

$$
\begin{aligned}
H= & \sum_{j=1,2,3, \ldots}\left[\xi\left(c_{2 j-1}^{*} c_{2 j}-c_{2 j-1} c_{2 j}^{*}\right)\right. \\
& \left.+\eta\left(c_{2 j}^{*} c_{2 j+1}-c_{2 j} c_{2 j+1}^{*}\right)\right] \\
& +\sum_{i=1,2,3, \ldots} \mu_{B} g^{*} B\left(\frac{1}{2}\right)\left(2 c_{i}^{*} c_{i}-1\right) .
\end{aligned}
$$

We exactly solve the eigenvalue problem of this Hamiltonian.

New operators $a_{j}$ and $b_{j}$ are introduced as follows:

$$
a_{j}=c_{2 j-1}, \quad b_{j}=c_{2 j}, \quad a_{j}^{*}=c_{2 j-1}^{*}, \quad b_{j}^{*}=c_{2 j}^{*},
$$


where $j$ is the cell number. Then the Hamiltonian (34) becomes

$$
\begin{aligned}
H= & \sum_{j=1}^{J}\left[\xi\left(a_{j}^{*} b_{j}-a_{j} b_{j}^{*}\right)+\eta\left(b_{j}^{*} a_{j+1}-b_{j} a_{j+1}^{*}\right)\right] \\
& +\sum_{j=1}^{J} \mu_{B} g^{*} B\left(\frac{1}{2}\right)\left(2 a_{j}^{*} a_{j}+2 b_{j}^{*} b_{j}-2\right),
\end{aligned}
$$

where $J$ is the total number of cells given by $J=N / 2$ for the total number of electrons $N$. We apply a Fourier transformation given by (38) to the operators $a_{j}, a_{j}^{*}, b_{j}$, and $b_{j}^{*}$ and obtain

$$
\begin{aligned}
H=\sum_{p}\left[\xi\left(a^{*}(p) b(p)+b^{*}(p) a(p)\right)\right. \\
\left.+\eta\left(e^{i p} b^{*}(p) a(p)+e^{-i p} a^{*}(p) b(p)\right)\right] \\
+\sum_{p} \mu_{B} g^{*} B\left(\frac{1}{2}\right)\left(2 a^{*}(p) a(p)+2 b^{*}(p) b(p)-2\right),
\end{aligned}
$$

where $a_{n}=\frac{1}{\sqrt{J}} \sum_{p} e^{i p n} a(p), \quad b_{n}=\frac{1}{\sqrt{J}} \sum_{p} e^{i p n} b(p)$,

and $p=(2 \pi / J) \times$ integer, $-\pi<p \leq \pi$.

In the summation in (37), the single term with a value of $p$ is expressed by the following matrix:

$$
\left(\begin{array}{cc}
\mu_{B} g^{*} B & \xi+\eta e^{-i p} \\
\xi+\eta e^{i p} & \mu_{B} g^{*} B
\end{array}\right)
$$

This matrix has two eigenvalues $\lambda_{1}(p)$ and $\lambda_{2}(p)$ which are given by

$$
\begin{aligned}
& \lambda_{1}(p)=\mu_{B} g^{*} B-\sqrt{\xi^{2}+\eta^{2}+2 \xi \eta \cos p} \\
& \lambda_{2}(p)=\mu_{B} g^{*} B+\sqrt{\xi^{2}+\eta^{2}+2 \xi \eta \cos p} .
\end{aligned}
$$

Using new annihilation operators $A_{1}(p)$ and $A_{2}(p)$

$$
\begin{aligned}
& A_{1}(p)=\frac{1}{\sqrt{2}} a(p)-\frac{\xi+\eta e^{-i p}}{\sqrt{2\left(\xi^{2}+\eta^{2}+2 \xi \eta \cos p\right)}} b(p), \\
& A_{2}(p)=\frac{1}{\sqrt{2}} a(p)+\frac{\xi+\eta e^{-i p}}{\sqrt{2\left(\xi^{2}+\eta^{2}+2 \xi \eta \cos p\right)}} b(p),
\end{aligned}
$$

the Hamiltonian (37) is expressed as follows:

$$
\begin{aligned}
H=\sum_{p}( & \lambda_{1}(p) A_{1}^{*}(p) A_{1}(p) \\
& \left.+\lambda_{2}(p) A_{2}^{*}(p) A_{2}(p)-\mu_{B} g^{*} B\right) .
\end{aligned}
$$

Thus, we have succeeded in diagonalizing the Hamiltonian (26).

\section{Magnetic Field Dependence of the Spin Polarization}

The electron spin polarization $\gamma_{e}$ depends upon the temperature. We calculate its thermodynamic mean value as follows:

$$
\begin{aligned}
\gamma_{e} & =\frac{1}{N}\left\langle-\sum_{i=1}^{N} \sigma_{i}^{z}\right\rangle=\frac{-1}{N}\left\langle\sum_{i=1}^{N}\left(2 c_{i}^{*} c_{i}-1\right)\right\rangle \\
& =\frac{-1}{N}\left\langle\sum_{j=1}^{J}\left(2 a_{j}^{*} a_{j}+2 b_{j}^{*} b_{j}-2\right)\right\rangle \\
& =\frac{-1}{2 J}\left\langle\sum_{p}\left(2 a^{*}(p) a(p)+2 b^{*}(p) b(p)-2\right)\right\rangle \\
& =\frac{-1}{2 J}\left\langle\sum_{p}\left(\sum_{s=1}^{2}\left(2 A_{s}^{*}(p) A_{s}(p)-1\right)\right)\right\rangle,
\end{aligned}
$$

where $\langle\cdot\rangle$ is the thermal average and the minus sign comes from the negative charge of an electron. In deriving (43), we have utilized (33c), (35), (38), (41a), and (41b). The diagonal form, (42), indicates that all the eigenenergy states are expressed by the direct product of the creation operators $A_{s}^{*}(p)$. Therefore, the eigenstate is identified by the eigenvalue of the fermion number operator $n_{s}(p)=A_{s}^{*}(p) A_{s}(p)$. Equation (42) shows that the eigenenergy for $n_{s}(p)=1$ is $\lambda_{s}(p)$ and the eigenenergy for $n_{s}(p)=0$ is zero. Then, the Boltzmann factor is $\exp \left(-\lambda_{s}(p) / k_{B} T\right)$ for $n_{s}(p)=1$ and $\exp \left(-0 / k_{B} T\right)$ for $n_{s}(p)=0$, where $k_{B}$ is the Boltzmann constant and $T$ is the temperature. Accordingly, the probability for $n_{s}(p)=1$ is given by $\exp \left(-\lambda_{s}(p) / k_{B} T\right) /(1+$ $\left.\exp \left(-\lambda_{s}(p) / k_{B} T\right)\right)$ and the probability for $n_{s}(p)=0$ is given by $1 /\left(1+\exp \left(-\lambda_{s}(p) / k_{B} T\right)\right)$. These probabilities yield the thermal average of $A_{s}^{*}(p) A_{s}(p)$ as

$$
\left\langle A_{s}^{*}(p) A_{s}(p)\right\rangle=\frac{\exp \left(-\lambda_{s}(p) / k_{B} T\right)}{1+\exp \left(-\lambda_{s}(p) / k_{B} T\right)},
$$

which gives

$$
\begin{aligned}
\left\langle 2 A_{s}^{*}(p) A_{s}(p)-1\right\rangle & =\frac{\exp \left(-\lambda_{s}(p) / k_{B} T\right)-1}{1+\exp \left(-\lambda_{s}(p) / k_{B} T\right)} \\
& =-\tanh \left(\frac{\lambda_{s}(p)}{\left(2 k_{B} T\right)}\right) .
\end{aligned}
$$

Substitution of (45) into (43) derives

$$
\gamma_{e}=\frac{1}{2 J} \sum_{p}\left(\sum_{s=1}^{2} \tanh \left(\frac{\lambda_{s}(p)}{2 k_{B} T}\right)\right) .
$$

Since the total number of electrons is a macroscopic value, we can replace the summation by integration as

$$
\gamma_{e}=\frac{1}{4 \pi} \int_{-\pi}^{\pi} \mathrm{d} p\left(\sum_{s=1}^{2} \tanh \left(\frac{\lambda_{s}(p)}{2 k_{B} T}\right)\right) .
$$




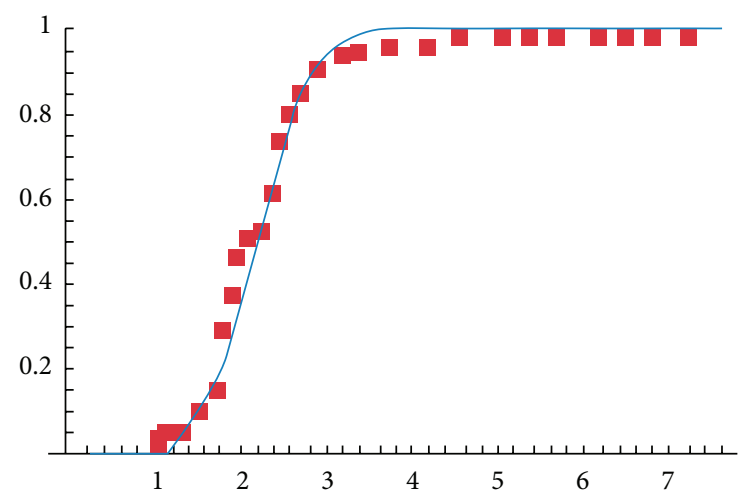

FIGURE 7: Calculated spin polarization curve for $v=2 / 3 \eta / \xi=$ $0.2 k_{B} T / \xi=0.1$. Red dots are experimental data [50].

Thus, the electron-spin polarization at the filling factor of $2 / 3$ is expressed by (47). tion.

First, we study the low field behavior of the spin polariza-

Equation (40a) indicates that $\lambda_{1}(p)$ is restricted to the following region:

$$
\mu_{B} g^{*} B-|\xi+\eta| \leq \lambda_{1}(p) \leq \mu_{B} g^{*} B-|\xi-\eta| .
$$

Also, $\lambda_{2}(p)$ is in the region:

$$
\mu_{B} g^{*} B+|\xi-\eta| \leq \lambda_{2}(p) \leq \mu_{B} g^{*} B+|\xi+\eta| .
$$

When the magnetic field takes a value between 0 and $|\xi-\eta| /\left(\mu_{B} g^{*}\right), \lambda_{1}(p)$ is negative and $\lambda_{2}(p)$ is positive for any value of $p$ :

$$
\lambda_{1}(p)<0, \quad \lambda_{2}(p)>0 \quad \text { for } 0<B<\frac{|\xi-\eta|}{\left(\mu_{B} g^{*}\right)} .
$$

Therefore, $\tanh \left(\lambda_{1}(p) / 2 k_{B} T\right)$ is nearly equal to -1 and $\tanh \left(\lambda_{2}(p) / 2 k_{B} T\right)$ is nearly equal to 1 at very low temperatures $(T \approx 0)$. Then, the spin polarization is almost zero because the summation in the right hand side of (47) is nearly equal to zero. When the magnetic field increases beyond the value $|\xi-\eta| /\left(\mu_{B} g^{*}\right)$, the spin polarization increases continuously until it reaches the maximum value of 1 . This behavior is in agreement with the experimental data in Figure 1.

When the quality of a quantum Hall device is bad, many random potentials are produced by the impurities and lattice defects. Then, the plateau in the Hall resistance curve is rounded at both ends. Also, the wide plateau in the polarization curve is rounded by the random potentials. The effect resembles that of the thermal vibration. Therefore, we include the random potential effect into the value of $T$.

The spin polarization is evaluated by integrating the right hand side of (47). The integration has been done by using a computer program. The result is shown in Figure 7 for the parameter $\eta / \xi=0.2$ and $k_{B} T / \xi=0.1$. Experimental data [50] are plotted by red dots in the figure. We find that the theoretical result reproduces the experimental data without the small shoulder.

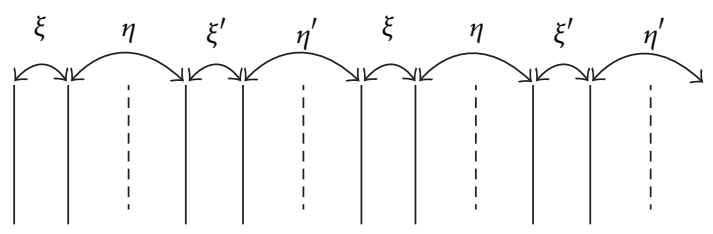

FIGURE 8: Coupling constants of interactions caused by distortion with double period.

\section{Modulation of the Intervals between Landau Orbitals}

If we observe carefully the experimental spin polarization curves, namely, Figure 1, we find small shoulders in it. The structure has not been considered in the previous sections. We examine the origin of the small shoulders in this section.

Peierls studied an electron system in a one-dimensional crystal and considered the lattice distortion with the period doubling the unit cell. This lattice distortion produces new band gaps and the energy becomes lower than that without the distortion. This effect is called spin Peierls effect [52].

In the present theory, the spin polarization of $\mathrm{FQH}$ states is derived from the Hamiltonians (26), (28), and so on. If we consider a new modulation of the intervals between the nearest Landau orbitals with the period doubling the unit configuration, the spin chain Hamiltonian of FQH system resembles the one with the spin Peierls effect.

As an example, for $v=2 / 3$ we change the distance between nearest orbitals in the first unit-configuration longer, the one in the second unit-configuration shorter, and so on. Then, we have the four kinds of the coupling constants $\xi, \xi^{\prime}$, $\eta$, and $\eta^{\prime}$ as shown in Figure 8.

The value of $\xi^{\prime}$ is larger than that of $\xi$ because the distance for the $\xi^{\prime}$ interaction path is shorter than that for $\xi$. Also, $\eta^{\prime}>$ $\eta$ holds. This distortion with the double period of the unitconfiguration produces additional energies via the classical Coulomb and spin exchange interactions. We call it "interval modulation." We calculate the total energy of this system.

We express the distance between the nearest orbitals by the symbol $r_{0}$ for nondistortion case. We consider the distortion that one of the distances between the nearest Landau orbitals becomes $r_{0}+d$ for an odd cell number and the other one becomes $r_{0}-d$ for an even cell number. Then, the classical Coulomb energy $W$ increases. The increasing value per electron is proportional to $d^{2}$ as

$$
\frac{\Delta W}{N}=f_{C}\left(\frac{d}{r_{0}}\right)^{2}
$$

where $f_{C}$ is the constant parameter.

Next, we examine $d$-dependence of the coupling constants $\xi$ and $\xi^{\prime}$. When $d>0$, the coupling constant $\xi$ is weaker than $\xi^{\prime}$ because the $\xi$ interaction path is longer than that of $\xi^{\prime}$. When the other case $d<0, \xi$ is stronger than $\xi^{\prime}$ because the 
$\xi$ interaction path becomes shorter than that of $\xi^{\prime}$. Therefore, there is a linear term of $d$ in $\xi$ and $\xi^{\prime}$ as follows:

$$
\xi=\xi_{0}-f_{\xi}\left(\frac{d}{r_{0}}\right), \quad \xi^{\prime}=\xi_{0}+f_{\xi}\left(\frac{d}{r_{0}}\right)
$$

where $\xi_{0}$ is the coupling constant in the non-distortion case and $f_{\xi}$ is the proportionality constant. In order to simplify (50) and (51), we define a new dimensionless quantity $t$ as

$$
t=\left(\frac{f_{\xi}}{\xi_{0}}\right)\left(\frac{d}{r_{0}}\right)
$$

Then the coupling constants $\xi$ and $\xi^{\prime}$ are expressed as

$$
\xi=\xi_{0}(1-t), \quad \xi^{\prime}=\xi_{0}(1+t) .
$$

The increasing value of the classical Coulomb energy $\Delta W$ is also expressed by this dimensionless parameter $t$ as follows:

$$
\frac{\Delta W}{N}=\xi_{0} C t^{2}
$$

where $C$ is the dimensionless coefficient as

$$
C=\frac{\xi_{0} f_{C}}{f_{\xi}^{2}}
$$

\section{Total Energy due to the Interval Modulation}

Now we calculate the spin exchange energy. Using the coupling constants in Figure 8, the spin exchange Hamiltonian for $v=2 / 3$ is given by

$$
\begin{aligned}
& H=\sum_{j=1,2,3, \ldots}\left[\xi\left(\sigma_{4 j-3}^{+} \sigma_{4 j-2}^{-}+\sigma_{4 j-3}^{-} \sigma_{4 j-2}^{+}\right)\right. \\
&+\left.\eta\left(\sigma_{4 j-2}^{+} \sigma_{4 j-1}^{-}+\sigma_{4 j-2}^{-} \sigma_{4 j-1}^{+}\right)\right] \\
&+\sum_{j=1,2,3, \ldots}\left[\xi^{\prime}\left(\sigma_{4 j-1}^{+} \sigma_{4 j}^{-}+\sigma_{4 j-1}^{-} \sigma_{4 j}^{+}\right)\right. \\
&\left.+\eta^{\prime}\left(\sigma_{4 j}^{+} \sigma_{4 j+1}^{-}+\sigma_{4 j}^{-} \sigma_{4 j+1}^{+}\right)\right] \\
&+\sum_{j=1,2,3, \ldots} \mu_{B} g^{*} B\left(\frac{1}{2}\right)\left(\sigma_{4 j-3}^{z}+\sigma_{4 j-2}^{z}+\sigma_{4 j-1}^{z}+\sigma_{4 j}^{z}\right) .
\end{aligned}
$$

This Hamiltonian equation (55) is rewritten from (33a), (33b), and (33c) as

$$
\begin{array}{r}
H=\sum_{j=1,2,3, \ldots}\left[\xi\left(c_{4 j-3}^{*} c_{4 j-2}-c_{4 j-3} c_{4 j-2}^{*}\right)\right. \\
\left.+\eta\left(c_{4 j-2}^{*} c_{4 j-1}-c_{4 j-2} c_{4 j-1}^{*}\right)\right] \\
+\sum_{j=1,2,3, \ldots}\left[\xi^{\prime}\left(c_{4 j-1}^{*} c_{4 j}-c_{4 j-1} c_{4 j}^{*}\right)\right. \\
\left.+\eta^{\prime}\left(c_{4 j}^{*} c_{4 j+1}-c_{4 j} c_{4 j+1}^{*}\right)\right] \\
+\sum_{i=1,2,3, \ldots} \mu_{B} g^{*} B\left(\frac{1}{2}\right)\left(2 c_{i}^{*} c_{i}-1\right) .
\end{array}
$$

Using the cell number $j$, we introduce new operators $a_{1, j}$, $a_{2, j}, a_{3, j}$, and $a_{4, j}$ as follows:

$$
a_{1, j}=c_{4 j-3}, \quad a_{2, j}=c_{4 j-2}, \quad a_{3, j}=c_{4 j-1}, \quad a_{4, j}=c_{4 j} .
$$

Fourier transformation yields

$$
\begin{array}{ll}
a_{1, j}=\frac{1}{\sqrt{J}} \sum_{p} e^{i p j} a_{1}(p), & a_{2, j}=\frac{1}{\sqrt{J}} \sum_{p} e^{i p j} a_{2}(p), \\
a_{3, j}=\frac{1}{\sqrt{J}} \sum_{p} e^{i p j} a_{3}(p), & a_{4, j}=\frac{1}{\sqrt{J}} \sum_{p} e^{i p j} a_{4}(p),
\end{array}
$$

where $J$ is the total number of unit cells (unit configurations), namely, $J=N / 4$, and $p=(2 \pi / J) \times$ integer $(-\pi<p \leq \pi)$. Substitution of (57) and (58) into (56) gives

$$
\begin{aligned}
& H=\sum_{p}[ \xi\left(a_{1}^{*}(p) a_{2}(p)+a_{2}^{*}(p) a_{1}(p)\right) \\
&+ \eta\left(a_{2}^{*}(p) a_{3}(p)+a_{3}^{*}(p) a_{2}(p)\right) \\
&+ \xi^{\prime}\left(a_{3}^{*}(p) a_{4}(p)+a_{4}^{*}(p) a_{3}(p)\right) \\
&\left.+\eta^{\prime}\left(e^{i p} a_{4}^{*}(p) a_{1}(p)+e^{-i p} a_{1}^{*}(p) a_{4}(p)\right)\right] \\
&+\sum_{p} \mu_{B} g^{*} B\left(\frac{1}{2}\right)\left(2 \left(a_{1}^{*}(p) a_{1}(p)+a_{2}^{*}(p) a_{2}(p)\right.\right. \\
&+a_{3}^{*}(p) a_{3}(p) \\
&\left.\left.+a_{4}^{*}(p) a_{4}(p)\right)-4\right) .
\end{aligned}
$$

For one value of $p$, (59) is expressed by the following matrix $M$ :

$$
M=\left(\begin{array}{cccc}
\mu_{B} g^{*} B & \xi & 0 & \eta^{\prime} e^{-i p} \\
\xi & \mu_{B} g^{*} B & \eta & 0 \\
0 & \eta & \mu_{B} g^{*} B & \xi^{\prime} \\
\eta^{\prime} e^{i p} & 0 & \xi^{\prime} & \mu_{B} g^{*} B
\end{array}\right) .
$$

The four eigenvalues of $M$ are denoted by the symbols $\lambda_{1}(p), \lambda_{2}(p), \lambda_{3}(p)$, and $\lambda_{4}(p)\left(\lambda_{1} \leq \lambda_{2} \leq \lambda_{3} \leq \lambda_{4}\right)$ which are given by 


$$
\begin{aligned}
& \lambda_{1}(p)=\mu_{B} g^{*} B-\sqrt{\frac{1}{2}\left(\left(1+\alpha^{2}\right)\left(\xi^{2}+\xi^{\prime 2}\right)+\sqrt{\left(1+\alpha^{2}\right)^{2}\left(\xi^{2}+\xi^{\prime 2}\right)^{2}-4\left(\left(1+\alpha^{4}\right) \xi^{2} \xi^{\prime 2}-2 \alpha^{2} \xi^{2} \xi^{\prime 2} \cos p\right)}\right)}, \\
& \lambda_{2}(p)=\mu_{B} g^{*} B-\sqrt{\frac{1}{2}\left(\left(1+\alpha^{2}\right)\left(\xi^{2}+\xi^{\prime 2}\right)-\sqrt{\left(1+\alpha^{2}\right)^{2}\left(\xi^{2}+\xi^{\prime 2}\right)^{2}-4\left(\left(1+\alpha^{4}\right) \xi^{2} \xi^{\prime 2}-2 \alpha^{2} \xi^{2} \xi^{\prime 2} \cos p\right)}\right)}, \\
& \lambda_{3}(p)=\mu_{B} g^{*} B+\sqrt{\frac{1}{2}\left(\left(1+\alpha^{2}\right)\left(\xi^{2}+\xi^{\prime 2}\right)-\sqrt{\left(1+\alpha^{2}\right)^{2}\left(\xi^{2}+\xi^{\prime 2}\right)^{2}-4\left(\left(1+\alpha^{4}\right) \xi^{2} \xi^{\prime 2}-2 \alpha^{2} \xi^{2} \xi^{\prime 2} \cos p\right)}\right)}, \\
& \lambda_{4}(p)=\mu_{B} g^{*} B+\sqrt{\frac{1}{2}\left(\left(1+\alpha^{2}\right)\left(\xi^{2}+\xi^{\prime 2}\right)+\sqrt{\left(1+\alpha^{2}\right)^{2}\left(\xi^{2}+\xi^{\prime 2}\right)^{2}-4\left(\left(1+\alpha^{4}\right) \xi^{2} \xi^{\prime 2}-2 \alpha^{2} \xi^{2} \xi^{\prime 2} \cos p\right)}\right)} .
\end{aligned}
$$

Therein, we have assumed $\xi^{\prime} / \xi=\eta^{\prime} / \eta$ because the interval modulation is expected to give almost the same effect to the coupling constants $\xi$ and $\eta$. Then, ratios between the coupling constants are expressed as follows:

$$
\begin{gathered}
\alpha=\frac{\eta}{\xi}=\frac{\eta^{\prime}}{\xi^{\prime}}=\frac{\eta_{0}}{\xi_{0}}, \\
\beta=\frac{\xi^{\prime}}{\xi}=\frac{\eta^{\prime}}{\eta} .
\end{gathered}
$$

Equation (53) derives the following relation:

$$
\beta=\frac{\xi^{\prime}}{\xi}=\frac{\eta^{\prime}}{\eta}=\frac{(1+t)}{(1-t)} .
$$

We show the two eigenvalues $\lambda_{1}(p)$ and $\lambda_{2}(p)$ by red and black curves for $\beta=1$, blue curves for $\beta=1.2$ and green curves for $\beta=1.4$ in Figure 9 .

The difference $\lambda_{2}(p)-\lambda_{1}(p)$ is minimal at $p=\pi$ as seen in Figure 9. When $\beta$ is equal to 1 , namely, nondistorted case, the energy gap $\lambda_{2}(\pi)-\lambda_{1}(\pi)$ disappears. Equation (61) gives the difference between $\lambda_{2}(p)$ and $\lambda_{1}(p)$ at $p=\pi$ as

$$
\lambda_{2}(\pi)-\lambda_{1}(\pi)=\sqrt{1+\alpha^{2}}\left|\xi^{\prime}-\xi\right|=\sqrt{1+\alpha^{2}} \xi_{0} 2 t
$$

where we have used (53). Thus, the difference is proportional to $t$.

The eigenenergies (61) give the diagonal form of the Hamiltonian as follows:

$$
\begin{aligned}
H=\sum_{p} & \left(\lambda_{1}(p) A_{1}^{*}(p) A_{1}(p)+\lambda_{2}(p) A_{2}^{*}(p) A_{2}(p)\right. \\
& +\lambda_{3}(p) A_{3}^{*}(p) A_{3}(p) \\
& \left.+\lambda_{4}(p) A_{4}^{*}(p) A_{4}(p)-2 \mu_{B} g^{*} B\right)
\end{aligned}
$$

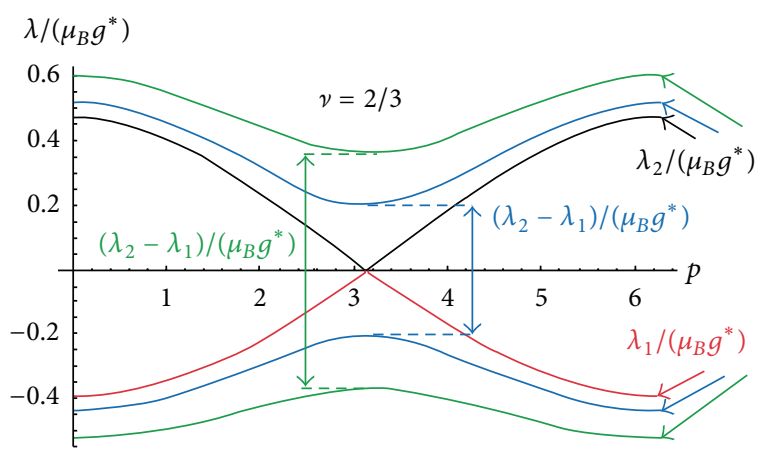

FIGURE 9: Eigenvalues of spin interaction via interval modulation. The red and black curves indicate the eigenvalues $\lambda_{1} /\left(\mu_{B} g^{*}\right)$ and $\lambda_{2} /\left(\mu_{B} g^{*}\right)$ at $\beta=1.0$. The blue curves indicate the eigenvalues $\lambda_{1} /\left(\mu_{B} g^{*}\right)$ and $\lambda_{2} /\left(\mu_{B} g^{*}\right)$ at $\beta=1.2$. The green curves indicate the eigenvalues $\lambda_{1} /\left(\mu_{B} g^{*}\right)$ and $\lambda_{2} /\left(\mu_{B} g^{*}\right)$ at $\beta=1.4$.

We calculate the thermal average of this Hamiltonian. The thermal average has been already examined in (44) which gives

$$
\begin{aligned}
\left\langle A_{s}^{*}(p) A_{s}(p)\right\rangle & =\frac{\exp \left(-\lambda_{s}(p) / k_{B} T\right)}{1+\exp \left(-\lambda_{s}(p) / k_{B} T\right)} \\
& =\frac{1}{2}\left(1-\tanh \left(\frac{\lambda_{s}(p)}{\left(2 k_{B} T\right)}\right)\right) .
\end{aligned}
$$

Then the thermal average of the spin exchange energy is

$$
\begin{gathered}
\langle H\rangle=\sum_{p}\left[\left\{\sum_{s=1}^{4}\left(\lambda_{s}(p) \frac{1}{2}\left(1-\tanh \left(\frac{\lambda_{s}(p)}{\left(2 k_{B} T\right)}\right)\right)\right)\right\}\right. \\
\left.-2 \mu_{B} g^{*} B\right] .
\end{gathered}
$$


Since the total number of electrons is a macroscopic value, we can replace the summation by integration as

$$
\begin{aligned}
\frac{\langle H\rangle}{N}=\frac{1}{4} & \\
\times \frac{1}{2 \pi} \int_{p=0}^{2 \pi}[ & \left\{\sum_{s=1}^{4}\left(\lambda_{s}(p) \frac{1}{2}\left(1-\tanh \left(\frac{\lambda_{s}(p)}{2 k_{B} T}\right)\right)\right)\right. \\
& \left.\left.-2 \mu_{B} g^{*} B\right\}\right] \mathrm{d} p, \\
\frac{\Delta E_{\text {Total }}}{N} & =\left(\frac{\Delta W}{N}\right)+\Delta\left(\frac{\langle H\rangle}{N}\right) .
\end{aligned}
$$

We numerically calculate the integration in (67a). The dashed red curve in Figure 10 shows the calculated result of the spin exchange energy for $B=2 \cdot 2[T]$. The dashed black curve shows the classical Coulomb energy for the parameter $C=0.5$ (see (54a), (54b)). Their sum $\Delta E_{\text {Total }} / N$ is the total energy which is expressed by the blue curve. Then, the total energy has a minimum at a nonzero $t$ as shown in Figure 10. Consequently, the interval modulation actually occurs.

Further calculations are carried out for various values of the magnetic field in the case of $C=0.5$. The results are shown in Figure 11. The interval modulation occurs in the region of $1.86[T]<B<2.51[T]$.

We calculate the total energy for another case $v=3 / 5$. The coupling constants are illustrated in Figure 12.

These coupling constants in Figure 12 produce the $v=3 / 5$ Hamiltonian which is described by the following matrix:

$$
\left.\begin{array}{cccccc}
\mu_{B} g^{*} B & \xi & 0 & 0 & 0 & \eta^{\prime} e^{-i p} \\
\xi & \mu_{B} g^{*} B & \eta & 0 & 0 & 0 \\
0 & \eta & \mu_{B} g^{*} B & \eta & 0 & 0 \\
0 & 0 & \eta & \mu_{B} g^{*} B & \xi^{\prime} & 0 \\
0 & 0 & 0 & \xi^{\prime} & \mu_{B} g^{*} B & \eta^{\prime} \\
\eta^{\prime} e^{i p} & 0 & 0 & 0 & \eta^{\prime} & \mu_{B} g^{*} B
\end{array}\right) .
$$

This matrix has the six eigenvalues $\lambda_{1}(p), \lambda_{2}(p), \lambda_{3}(p)$, $\lambda_{4}(p), \lambda_{5}(p)$, and $\lambda_{6}(p)$, the $p$-dependences of which are shown in Figure 13.

Figure 13 indicates the energy gap between $\lambda_{1}(p)$ and $\lambda_{2}(p)$ at $p=\pi$. Then the interval modulation with the double period of the unit-configuration produces additional energies for the classical-Coulomb and spin-exchange interactions, respectively, as follows:

$$
\frac{\Delta W}{N}=\xi_{0} C t^{2},
$$

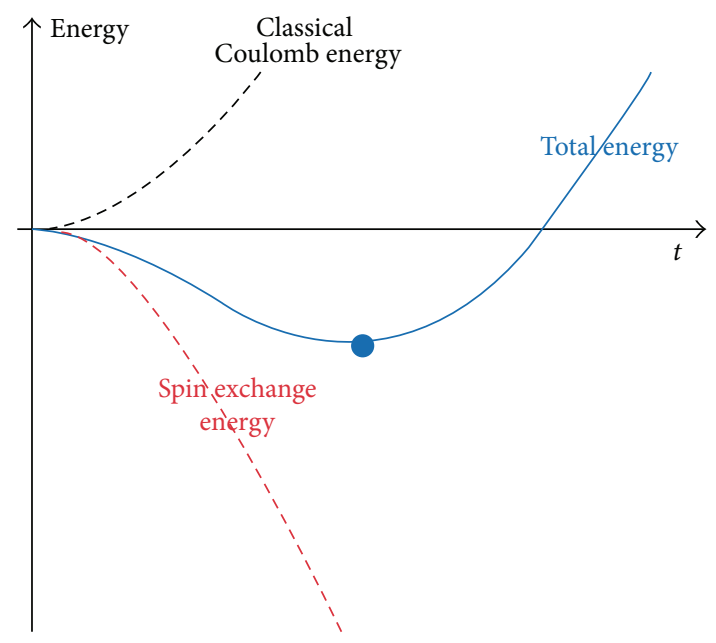

Figure 10: Dependence of total energy upon $t$.

$$
\begin{aligned}
\frac{\langle H\rangle}{N}=\frac{1}{6} & \\
\times \frac{1}{2 \pi} \int_{p=0}^{2 \pi}[ & \left\{\sum_{s=1}^{6}\left(\lambda_{s}(p) \frac{1}{2}\left(1-\tanh \left(\frac{-\lambda_{s}(p)}{2 k_{B} T}\right)\right)\right)\right) \\
& \left.\left.-3 \mu_{B} g^{*} B\right\}\right] \mathrm{d} p \\
\frac{\Delta E_{\text {Total }}}{N} & =\left(\frac{\Delta W}{N}\right)+\Delta\left(\frac{\langle H\rangle}{N}\right)
\end{aligned}
$$

The sum of $\Delta W / N$ and $\Delta(\langle H\rangle / N)$ is numerically calculated and the result is shown in Figure 14.

Therein, we have used the parameter $C=0.5$ which is the same as in the case of $v=2 / 3$. This value of $C$ affects the shape of the polarization curve near the small shoulder as will be discussed in the next section. We have shown the dependence of the total energy upon $t$ for two examples. Therein, the total energy has a minimum at a nonzero $t$ in some region, of the magnetic field. The nonzero $t$ yields the stable state with the distortion (interval modulation). This mathematical mechanism is the one resembling the spin Peierls effect.

\section{Spin Polarization in the Case with the Interval Modulation}

We calculate the spin-polarization $\gamma_{e}$ for the Hamiltonian (64) of $v=2 / 3 \cdot \gamma_{e}$ is obtained by the integration as

$$
\gamma_{e}=\frac{1}{4} \times \frac{1}{2 \pi} \int_{-\pi}^{\pi} \mathrm{d} p\left(\sum_{s=1}^{4} \tanh \left(\frac{\lambda_{s}(p)}{2 k_{B} T}\right)\right),
$$

where the four eigen-values $\lambda_{1}(p), \lambda_{2}(p), \lambda_{3}(p)$, and $\lambda_{4}(p)$ are given in (61). We numerically calculate the spinpolarization $\gamma_{e}$ by the following two methods, namely, easy method A and precise method B. Method A is the rough calculation under the fixed value of the distortion parameter $t$. This method has been studied in the previous papers $[53,54]$ 


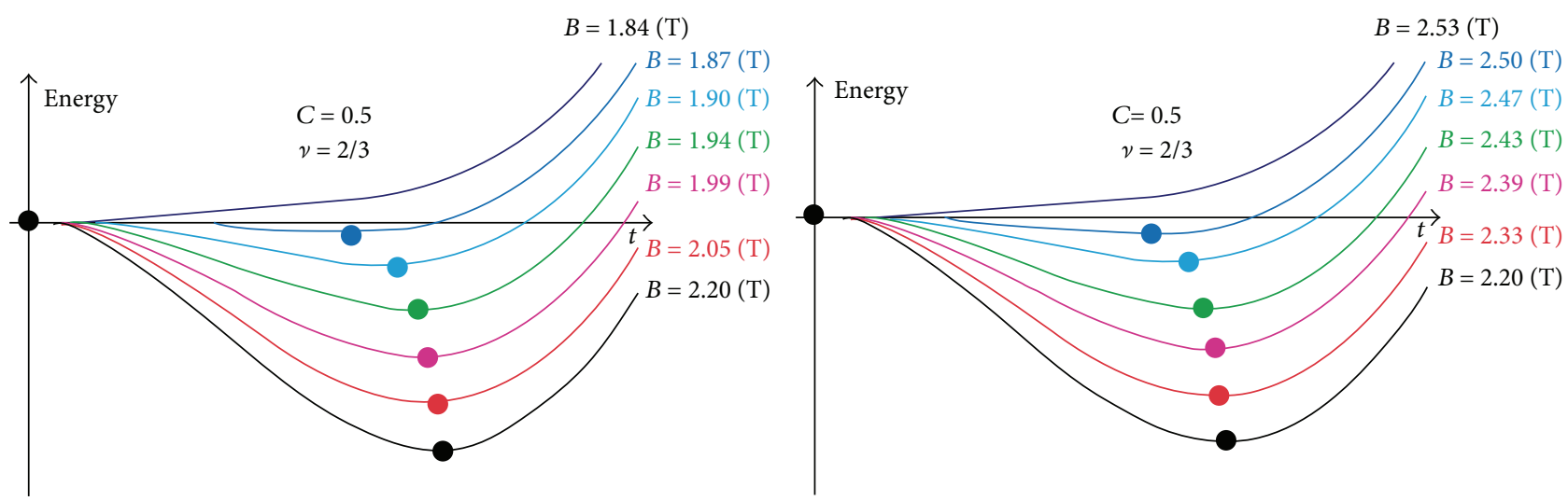

FIgURE 11: Dependence of total energy upon $t$.

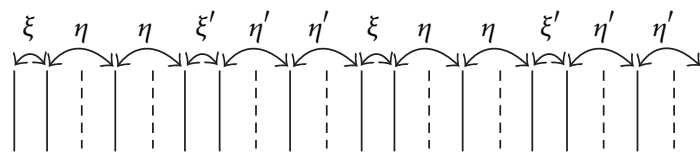

FIGURE 12: Coupling constants for $v=3 / 5$.

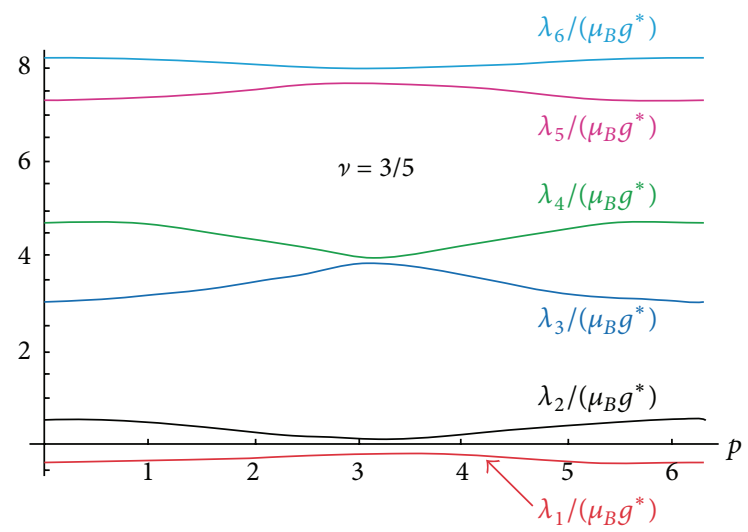

Figure 13: $p$-Dependence of the six eigen values for $v=3 / 5$.

where the ratio $\xi^{\prime} / \xi=\eta^{\prime} / \eta$ is treated to be a constant value as in (62c). Method B is newly performed in this paper.

Method A. We use the fixed value $\xi^{\prime} / \xi=\eta^{\prime} / \eta=1.4$. The other parameters are adopted to be $\eta / \xi=\eta^{\prime} / \xi^{\prime}=0.1$ and $\left(k_{B} T / \xi_{0}\right)=0.05$. Then, a small energy gap appears between $\lambda_{1}$ and $\lambda_{2}$. We numerically calculate the integration in (70) by using the Mathematica program and draw the graph of spinpolarization versus magnetic field. A small shoulder appears in the theoretical curve of the electron spin-polarization as seen in Figure 15.

This curve is slightly different from the experimental data near the sharp corners $P$ and $Q$. So we choose the different value as $\left(k_{B} T / \xi_{0}\right)=0.1$ in order to make the curvature small in the corners $P$ and $Q$. Then the small shoulder disappears. In order to maintain the small shoulder, we take a larger value $\xi^{\prime} / \xi=\eta^{\prime} / \eta=1.8$. The calculated curve is shown in
Figure 16. The result is also different from the experimental data. Accordingly, method A has some difficulty in explaining the experimental data. This inadequacy is improved by using the precise method $\mathrm{B}$.

Method B. We carry out more precise method B where we calculate the $t$-dependence of the total energy per electron. We find the minimum point of the total energy for various values of the magnetic field. Some examples have been already shown in Figures 11 and 14. Therein, we obtain the $t$-value of the minimum point. The $t$-value gives $\xi^{\prime} / \xi=\eta^{\prime} / \eta$ from $(62 c)$. We numerically calculate the spin-polarization by using the magnetic field dependence of the minimum point. The theoretical curve is shown in Figure 17.

Therein, we have used the parameter values $\eta / \xi=\eta^{\prime} / \xi^{\prime}=$ $0.2,\left(k_{B} T / \xi_{0}\right)=0.1$ and $C=0.5$. Thus, the calculated result is in good agreement with the experimental data. The reason is simply discussed below.

The magnetic field is sufficiently strong near the corner $P$ in Figure 15. In this region, almost all the spins have a down direction. Then the number of up- and downspin pairs decreases and the spin exchange energy becomes small. So the increment of the total energy (67b) is nearly equal to that of the classical Coulomb energy. Then, the energy minimum appears at $t=0$, namely, non-distortion (noninterval modulation). Thus, the distortion appears only near the small shoulder as in Figure 11. Due to this situation, the theoretical curve via method B is in good agreement with the experimental data.

It is examined how the shape of the polarization curve depends on the parameter $C$. We calculate the polarization curve for the following two cases: $C=0.4$ and 0.65 in Figure 18. These calculations make it clear that the shape of the curve varies only in the neighborhood of the small shoulder when changing the parameter $C$.

We study the case of $\nu=3 / 5$. The spin-polarization $\gamma_{e}$ for $\nu=3 / 5$ is given by

$$
\gamma_{e}=\frac{1}{6} \times \frac{1}{2 \pi} \int_{-\pi}^{\pi} \mathrm{d} p\left(\sum_{s=1}^{6} \tanh \left(\frac{\lambda_{s}(p)}{2 k_{B} T}\right)\right),
$$




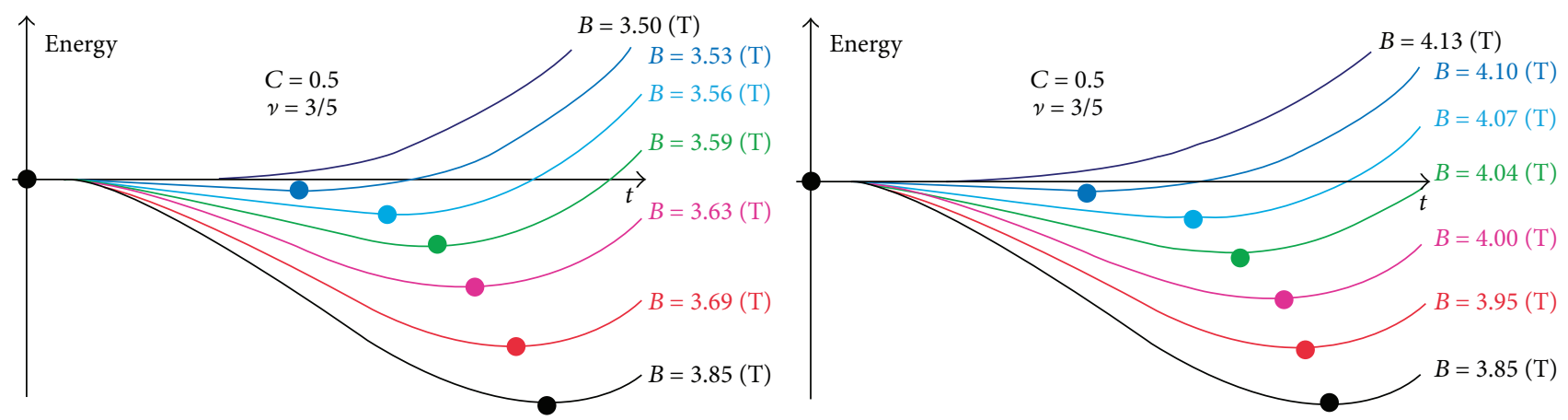

Figure 14: Dependence of the total energy upon $t$ at $v=3 / 5$.

where the eigen-energies $\lambda_{s}$ for $s=1,2,3,4,5,6$ are numerically obtained from the matrix (68). The two calculated curves via methods $A$ and $B$ are shown in Figure 19. In the method B we have applied $C=0.5$, same as in $v=2 / 3$. As seen in the right panel of Figure 19, the theoretical result via method B is in good agreement with the experimental data.

We next examine the case of $v=4 / 7$. The most uniform electron configuration is illustrated in Figure 20.

The electron configuration yields the $v=4 / 7$ Hamiltonian described by the following matrix (72):

$$
\left(\begin{array}{cccccccc}
\mu_{B} g^{*} B & \xi & 0 & 0 & 0 & 0 & 0 & \eta^{\prime} e^{-i p} \\
\xi & \mu_{B} g^{*} B & \eta & 0 & 0 & 0 & 0 & 0 \\
0 & \eta & \mu_{B} g^{*} B & \eta & 0 & 0 & 0 & 0 \\
0 & 0 & \eta & \mu_{B} g^{*} B & \eta & 0 & 0 & 0 \\
0 & 0 & 0 & \eta & \mu_{B} g^{*} B & \xi^{\prime} & 0 & 0 \\
0 & 0 & 0 & 0 & \xi^{\prime} & \mu_{B} g^{*} B & \eta^{\prime} & 0 \\
0 & 0 & 0 & 0 & 0 & \eta^{\prime} & \mu_{B} g^{*} B & \eta^{\prime} \\
\eta^{\prime} e^{i p} & 0 & 0 & 0 & 0 & 0 & \eta^{\prime} & \mu_{B} g^{*} B
\end{array}\right), \quad\left(\text { for } v=\frac{4}{7}\right)
$$

The spin polarization $\gamma_{e}$ is given by

$$
\gamma_{e}=\frac{1}{8} \times \frac{1}{2 \pi} \int_{-\pi}^{\pi} \mathrm{d} p\left(\sum_{s=1}^{8} \tanh \left(\frac{\lambda_{s}(p)}{2 k_{B} T}\right)\right) .
$$

Therein, the eight values $\lambda_{s}$ for $s=1,2,3,4,5,6,7,8$ indicate the eigenenergies of the matrix (72).

The spin polarization can be evaluated from the eigenenergies. The results are shown in Figure 21. Method B has used the same value 0.5 for the parameter $C$.

\section{Effect Resembling Spin Peierls One in Various Filling Factors}

We examine the cases of $v=2 / 5, v=3 / 7$, and $v=$ $4 / 9$ with the interval modulation, the most uniform electron configurations of which are illustrated in Figures 22(a), 22(b), and $22(\mathrm{c})$, respectively.

These electron configurations and their coupling constants yield the spin-exchange Hamiltonians for $v=2 / 5$, $\nu=3 / 7$, and $v=4 / 9$, which are represented by the following matrices, respectively:

$$
\begin{aligned}
& \left(\begin{array}{cccc}
\mu_{B} g^{*} B & \eta & 0 & \varsigma^{\prime} e^{-i p} \\
\eta & \mu_{B} g^{*} B & \varsigma & 0 \\
0 & \varsigma & \mu_{B} g^{*} B & \eta^{\prime} \\
\varsigma^{\prime} e^{i p} & 0 & \eta^{\prime} & \mu_{B} g^{*} B
\end{array}\right), \quad\left(\text { for } v=\frac{2}{5}\right) \\
& \left(\begin{array}{cccccc}
\mu_{B} g^{*} B & \eta & 0 & 0 & 0 & \varsigma^{\prime} e^{-i p} \\
\eta & \mu_{B} g^{*} B & \eta & 0 & 0 & 0 \\
0 & \eta & \mu_{B} g^{*} B & \varsigma & 0 & 0 \\
0 & 0 & \varsigma & \mu_{B} g^{*} B & \eta^{\prime} & 0 \\
0 & 0 & 0 & \eta^{\prime} & \mu_{B} g^{*} B & \eta^{\prime} \\
\varsigma^{\prime} e^{i p} & 0 & 0 & 0 & \eta^{\prime} & \mu_{B} g^{*} B
\end{array}\right), \quad\left(\text { for } v=\frac{3}{7}\right)
\end{aligned}
$$




$$
\left.\left(\begin{array}{cccccccc}
\mu_{B} g^{*} B & \eta & 0 & 0 & 0 & 0 & 0 & \varsigma^{\prime} e^{-i p} \\
\eta & \mu_{B} g^{*} B & \eta & 0 & 0 & 0 & 0 & 0 \\
0 & \eta & \mu_{B} g^{*} B & \eta & 0 & 0 & 0 & 0 \\
0 & 0 & \eta & \mu_{B} g^{*} B & \varsigma & 0 & 0 & 0 \\
0 & 0 & 0 & \varsigma & \mu_{B} g^{*} B & \eta^{\prime} & 0 & 0 \\
0 & 0 & 0 & 0 & \eta^{\prime} & \mu_{B} g^{*} B & \eta^{\prime} & 0 \\
0 & 0 & 0 & 0 & 0 & \eta^{\prime} & \mu_{B} g^{*} B & \eta^{\prime} \\
\varsigma^{\prime} e^{i p} & 0 & 0 & 0 & 0 & 0 & \eta^{\prime} & \mu_{B} g^{*} B
\end{array}\right), \quad \text { for } v=\frac{4}{9}\right)
$$

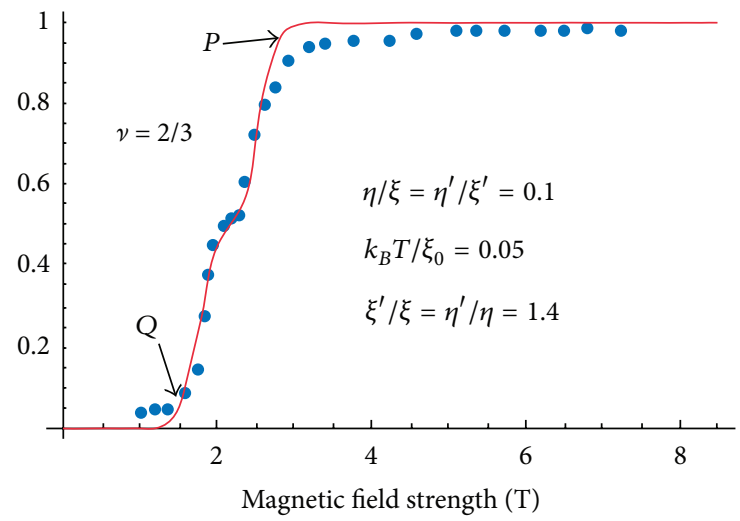

FIGURE 15: Method A: theoretical curve of the spin polarization for $\nu=2 / 3$. Blue dots are experimental data [50].

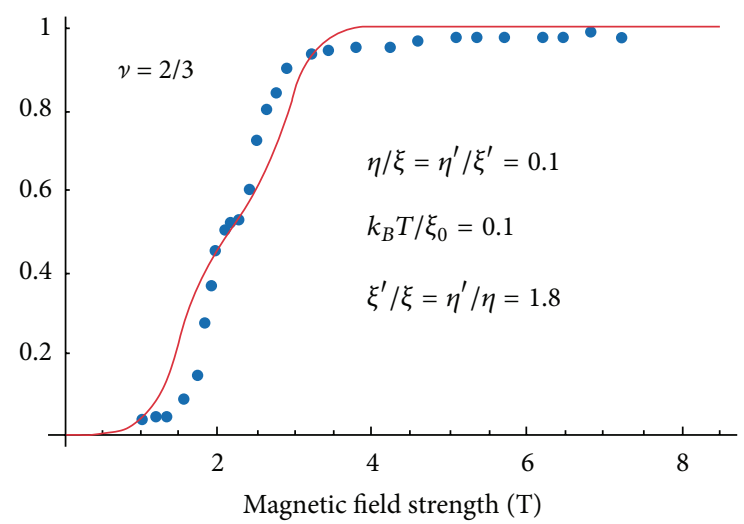

FIGURE 16: Method A: calculated curve of the spin polarization for $v=2 / 3$ (the parameters are chosen as $\left(k_{B} T / \xi_{0}\right)=0.1$ and $\xi^{\prime} / \xi=$ $\left.\eta^{\prime} / \eta=1.8\right)$.

The average value of $\xi$ and $\xi^{\prime}$ is equal to $\xi_{0}$. Also $\eta_{0}=$ $\left(\eta+\eta^{\prime}\right) / 2$. Accordingly, we obtain

$$
\eta=\eta_{0}(1-t), \quad \eta^{\prime}=\eta_{0}(1+t) .
$$

The ratios between the coupling constants satisfy the following relations:

$$
\begin{gathered}
\frac{\varsigma}{\eta}=\frac{\varsigma^{\prime}}{\eta^{\prime}}=\frac{\varsigma_{0}}{\eta_{0}}, \\
\frac{\eta^{\prime}}{\eta}=\frac{\varsigma^{\prime}}{\varsigma}=\frac{(1+t)}{(1-t)} .
\end{gathered}
$$

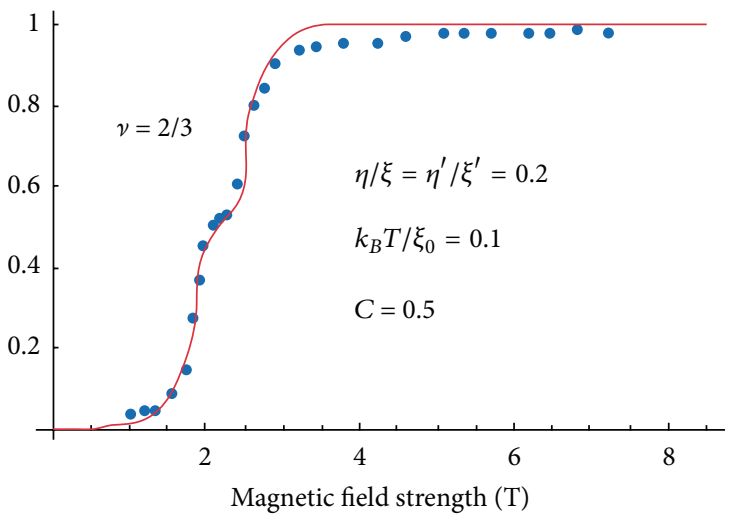

FIGURE 17: Method B: calculated curve of the spin-polarization for $\nu=2 / 3$.

In order to compare the spin exchange and classical Coulomb energies, we reexpress the $t$-dependence of the classical Coulomb energy by using the coupling constant $\eta_{0}$,

$$
\frac{\Delta W}{N}=\eta_{0} D t^{2}
$$

where $D$ is a new coefficient. Using the eigenvalues of the matrices, the spin polarization is given by

$$
\begin{array}{ll}
\gamma_{e}=\frac{1}{4} \times \frac{1}{2 \pi} \int_{-\pi}^{\pi} \mathrm{d} p\left(\sum_{s=1}^{4} \tanh \left(\frac{\lambda_{s}(p)}{2 k_{B} T}\right)\right), & \left(\text { for } v=\frac{2}{5}\right), \\
\gamma_{e}=\frac{1}{6} \times \frac{1}{2 \pi} \int_{-\pi}^{\pi} \mathrm{d} p\left(\sum_{s=1}^{6} \tanh \left(\frac{\lambda_{s}(p)}{2 k_{B} T}\right)\right), & \left(\text { for } v=\frac{3}{7}\right),
\end{array}
$$

$$
\gamma_{e}=\frac{1}{8} \times \frac{1}{2 \pi} \int_{-\pi}^{\pi} \mathrm{d} p\left(\sum_{s=1}^{8} \tanh \left(\frac{\lambda_{s}(p)}{2 k_{B} T}\right)\right), \quad\left(\text { for } v=\frac{4}{9}\right) .
$$

We numerically calculate the spin-polarization curves via method B, the results of which are shown in Figure 23.

It is found in these figures that the small shoulders originate from the interval modulation (distortion with double period). The theoretical curve via method $B$ is in better agreement with the experimental data than the one via method A. Here we shortly discuss the parameter values $C$ and $D$. The increasing value of the classical Coulomb 

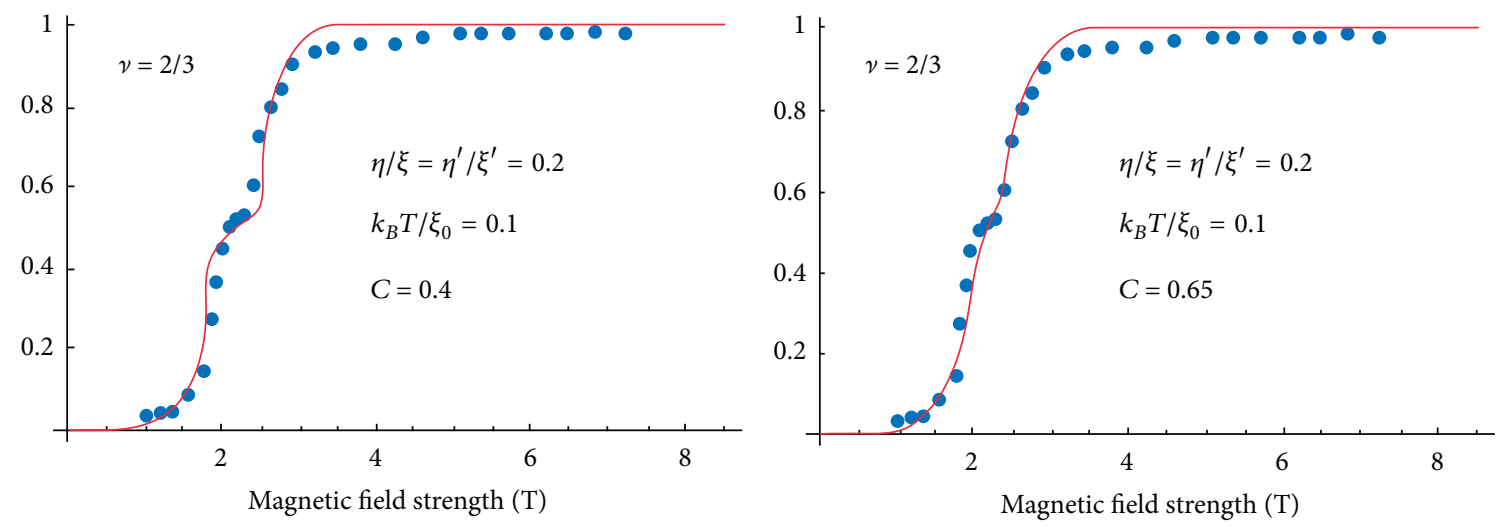

FIGURE 18: Spin-polarization via method B for two cases with $C=0.4$ and 0.65 .

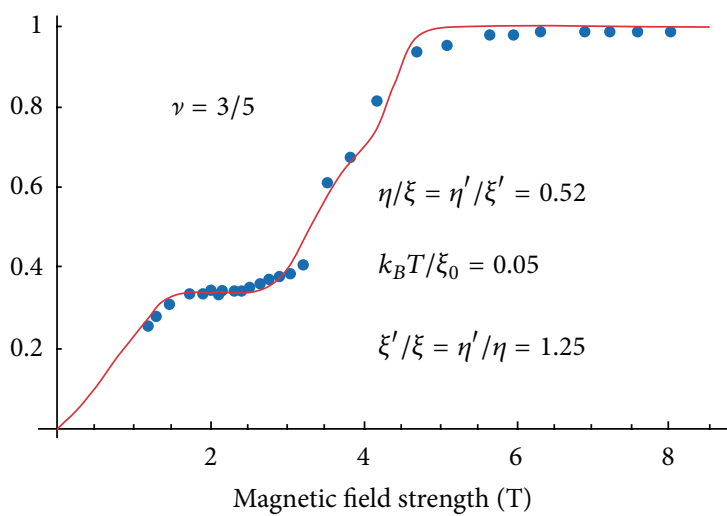

(Method A)

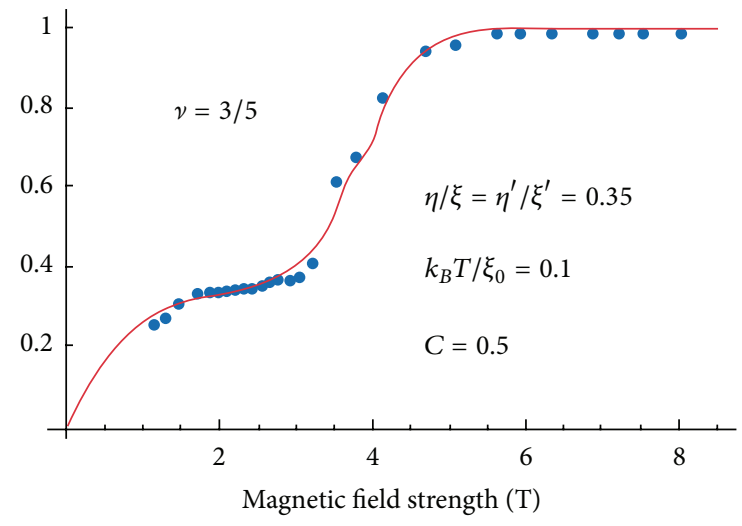

(Method B)

FIGURE 19: Spin-polarization for $\nu=3 / 5$. Blue dots are experimental data [50].

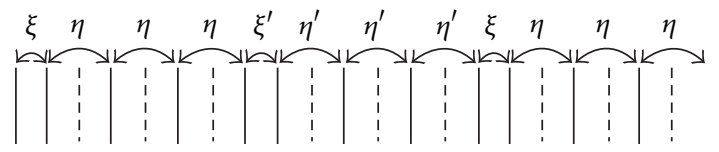

FIGURE 20: Coupling constants for $v=4 / 7$.

energy is expressed in (69a) and (77) as $\Delta W / N=\xi_{0} C t^{2}$ and $\Delta W / N=\eta_{0} D t^{2}$, respectively. So the parameter $D$ may be almost equal to

$$
D \approx C \times \frac{\xi_{0}}{\eta_{0}}
$$

The fitting values of $D$ are $0.65,1.5$, and 1.2 for $v=2 / 5$, $3 / 7$, and $4 / 9$, respectively, as shown in Figure 23 . We cannot understand why the parameter $D$ is small at $\nu=2 / 5$.

We examine the remaining cases of $\nu=3 / 7$ and $4 / 9$. The ratio $\eta / \xi=\eta^{\prime} / \xi^{\prime}=\eta_{0} / \xi_{0}$ is 0.35 for $\nu=3 / 5$ and $4 / 7$. Substituting this value 0.35 for $\eta_{0} / \xi_{0}$ into (79a) we obtain the value $D$ as

$$
D \approx \frac{0.5}{0.35} \approx 1.43
$$

which is the predicted value from the cases $v=3 / 5$ and $4 / 7$. The fitting values of $D$ are 1.5 and 1.2 for $v=3 / 7$ and $4 / 9$ as seen in Figure 23. These fitting values are consistent with 1.43 derived from the cases of $v=3 / 5$ and $4 / 7$.

Next, we examine the spin polarization at $v=4 / 3,7 / 5$, and $8 / 5$. The most uniform electron-configuration and the coupling constants are shown in Figures 24(a), 24(b), and $24(\mathrm{c})$, respectively.

There are doubly occupied orbitals in these electron configurations of Figures 24(a), 24(b), and 24(c). The spin exchange forces act between electrons in singly occupied orbitals. The electron pairs in doubly occupied orbitals have no polarization because of cancellation by up- and down-spin pairs. Therefore, the electron spin polarization is given by the following equations:

$$
\begin{array}{r}
\gamma_{e}=\frac{2}{4} \times \frac{1}{4} \times \frac{1}{2 \pi} \int_{-\pi}^{\pi} \mathrm{d} p\left(\sum_{s=1}^{4} \tanh \left(\frac{\lambda_{s}(p)}{2 k_{B} T}\right)\right), \\
\left(\text { for } \nu=\frac{4}{3}\right), \\
\gamma_{e}=\frac{3}{7} \times \frac{1}{6} \times \frac{1}{2 \pi} \int_{-\pi}^{\pi} \mathrm{d} p\left(\sum_{s=1}^{6} \tanh \left(\frac{\lambda_{s}(p)}{2 k_{B} T}\right)\right), \\
\left(\text { for } \nu=\frac{7}{5}\right),
\end{array}
$$




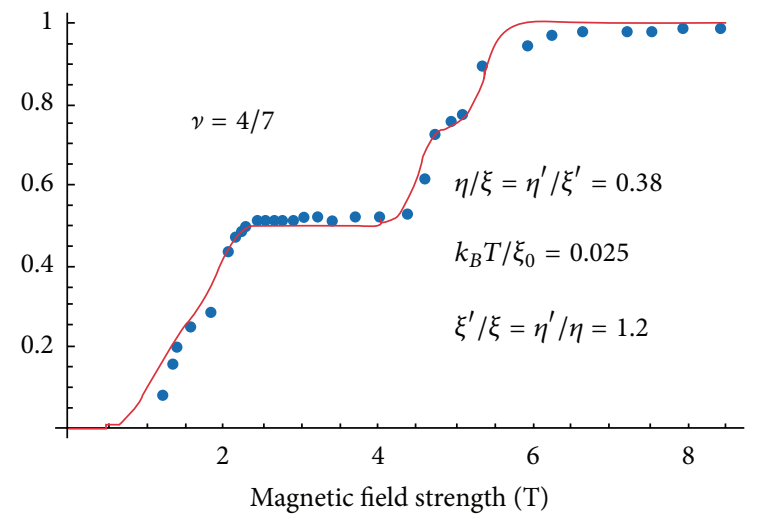

$($ Method A)

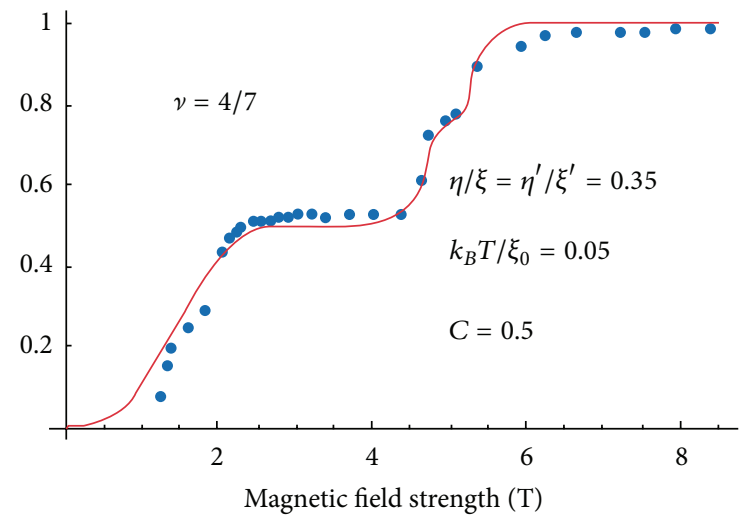

(Method B)

FIGURE 21: Spin-polarization for $\nu=4 / 7$. Blue dots are experimental data [50].

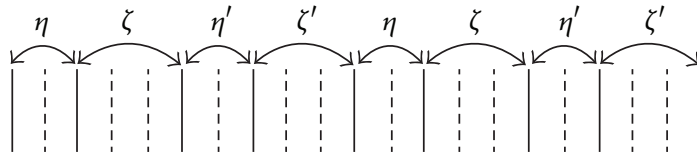

(a)

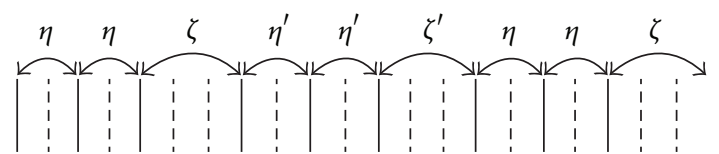

(b)

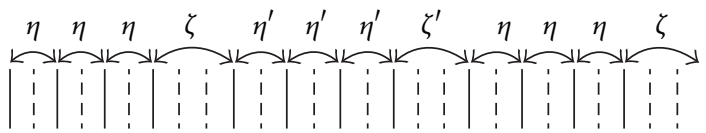

(c)

FIGURE 22: (a) Coupling constants for $v=2 / 5$. (b) Coupling constants for $v=3 / 7$. (c) Coupling constants for $v=4 / 9$.

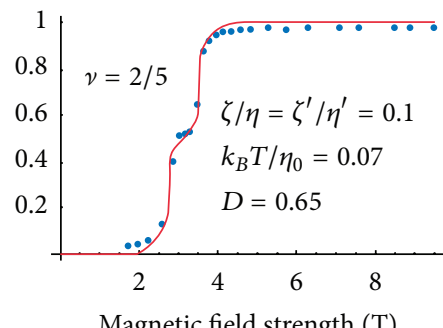

Magnetic field strength $(\mathrm{T})$

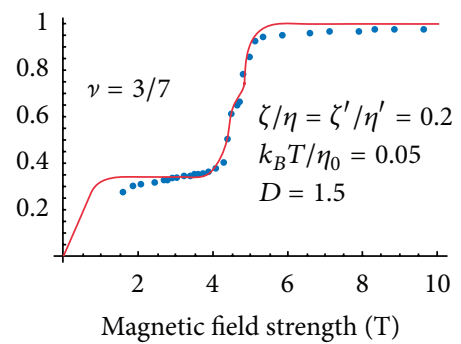

Magnetic field strength (T)

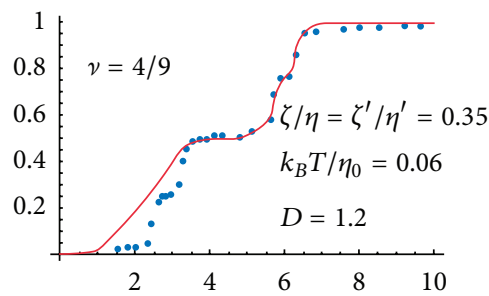

Magnetic field strength (T)

FIGURE 23: Spin-polarization for $v=2 / 5,3 / 7,4 / 9$. Blue dots are experimental data [50].

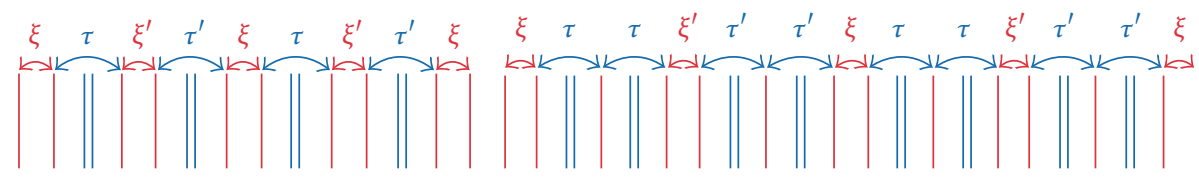

(a)

(b)

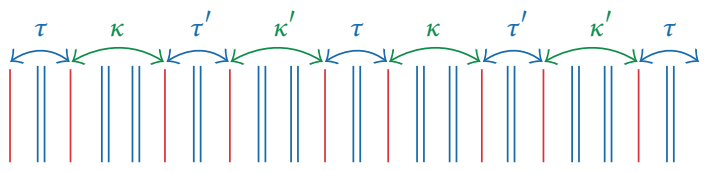

(c)

FIGURE 24: (a) Coupling constants for $v=4 / 3$. Double line indicates a Landau orbital occupied by an electron pair with up- and down-spins. (b) Coupling constants for $v=7 / 5$. Double line indicates a Landau orbital occupied by an electron pair with up-and down-spins. (c) Coupling constants for $v=8 / 5$. Double line indicates a Landau orbital occupied by an electron pair with up- and down-spins. 

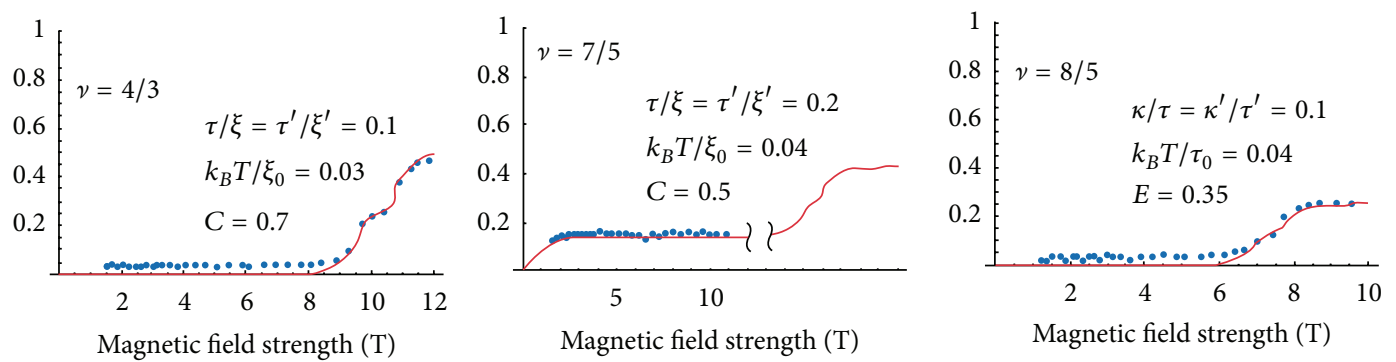

FIGURE 25: Spin-polarization for $v=4 / 3,7 / 5$, and 8/5. Blue dots are experimental data [50].

$$
\begin{array}{r}
\gamma_{e}=\frac{2}{8} \times \frac{1}{4} \times \frac{1}{2 \pi} \int_{-\pi}^{\pi} \mathrm{d} p\left(\sum_{s=1}^{4} \tanh \left(\frac{\lambda_{s}(p)}{2 k_{B} T}\right)\right), \\
\left(\text { for } \nu=\frac{8}{5}\right),
\end{array}
$$

where the coefficients $2 / 4,3 / 7$, and $2 / 8$ in (80), (81) and (82) come from the fact that the up- and down-spin pairs cancel the polarization. That is to says, two electrons per four electrons at $v=4 / 3$ have no polarization, four electrons per seven electrons at $v=7 / 5$ have no polarization, and also six electrons per eight electrons at $v=8 / 5$ have no polarization.

We numerically calculate the spin-polarization curves via method B, the results of which are shown in Figure 25.

The polarization data at $v=4 / 3$ has a very sharp change of the curvature from $B=8.5[T]$ to $B=11.5[T]$. Therefore, it is very difficult for the function to fit the experimental data. The theoretical result via method B is in good agreement with the experimental data at $v=4 / 3$, as seen in the left panel of Figure 25.

The coupling constants at $\nu=8 / 5$ are $\tau, \kappa, \tau^{\prime}, \kappa^{\prime}$ as in Figure 24(c). Accordingly, the coupling constants and the classical Coulomb energy are reexpressed by using $\tau_{0}$ as follows:

$$
\begin{gathered}
\tau=\tau_{0}(1-t), \quad \tau^{\prime}=\tau_{0}(1+t), \\
\frac{\Delta W}{N}=\tau_{0} E t^{2},
\end{gathered}
$$

where $E$ is a new coefficient. The fitting value is $E=0.35$ for $v=8 / 5$. This value is different from $C$ and $D$. Probably the reason is the shielding effect of the electron pair in doubly occupied Landau orbitals in Figure 24(c).

In the case of $v=4 / 3$ and $v=7 / 5$, the fitting values of $C$ are 0.7 and 0.5 , respectively. These values are nearly equal to $C=0.5$ in $v=2 / 3,3 / 5$, and $4 / 7$. The parameters $\eta / \xi,\left(k_{B} T / \xi_{0}\right), C$ and others may be dependent upon the gate voltage, sample, temperature, and so on. We have used almost same value for $C$. If we use different values of $C$, we can find a better fitting to the experimental data than the present results. As seen in Figures 17, 19, 21, 23, and 25, the small shoulders are caused by the interval modulation which comes from the Peierls instability.

\section{Conclusions}

(1) The $v=2 / 3$ polarization curve resembles the $v=2 / 5$ curve, the $v=3 / 5$ one resembles the $v=3 / 7$ one, and the $v=4 / 7$ one resembles the $v=4 / 9$ one. Thus, the shape of the spin polarization curves depends mainly upon the numerator of the filling factor. Therefore, the polarization belongs to electrons (not holes).

(2) At low field, up- and down-spins coexist. Then, there are many degenerate ground states which are composed of different spin arrangements for a given electron-configuration in the Landau orbitals. These many electron states have the same eigenenergy of $H_{D}$. We have succeeded to diagonalize the partial Hamiltonian describing the Coulomb transitions among the degenerate ground states. The calculated results reproduce the wide plateaus in the spin polarization curves of the experimental data [50].

(3) The experimental curve of the polarization versus magnetic field exhibits small shoulders. These small shoulders are derived from the following mechanism. We have exactly solved the partial Hamiltonian and also have minimized the total energy (sum of the spin exchange and classical Coulomb energies). The total energy decreases by modulating the intervals between Landau orbitals with the doubly period. Calculating the $t$-value with the minimum energy, we have found that the interval modulation actually occurs. Then, the theoretical polarization curve reproduces the small shoulder and the wide plateau. The results of the present theory are in good agreement with the experimental data.

\section{Acknowledgments}

The author expresses his heartfelt appreciation for the encouragement of Professor Koichi Katsumata, Professor Hidenobu Hori, Professor Yasuyuki Kitano, and Professor Masayuki Hagiwara. Particularly Professor Katsumata has given the author the important suggestions for improving my description of this paper. 


\section{References}

[1] W. Pan, H. L. Stormer, D. C. Tsui, L. N. Pfeiffer, K. W. Baldwin, and K. W. West, "Transition from an electron solid to the sequence of fractional quantum Hall states at very low Landau level filling factor," Physical Review Letters, vol. 88, no. 17, Article ID 176802, 4 pages, 2002.

[2] W. Pan, H. L. Stormer, D. C. Tsui, L. N. Pfeiffer, K. W. Baldwin, and K. W. West, "Fractional quantum Hall effect of composite fermions," Physical Review Letters, vol. 90, no. 1, Article ID 016801, 4 pages, 2003.

[3] R. B. Laughlin, "Quantized motion of three two-dimensional electrons in a strong magnetic field," Physical Review B, vol. 27, no. 6, pp. 3383-3389, 1983.

[4] R. B. Laughlin, "Anomalous quantum Hall effect: an incompressible quantum fluid with fractionally charged excitations," Physical Review Letters, vol. 50, no. 18, pp. 1395-1398, 1983.

[5] F. D. M. Haldane, "Fractional quantization of the Hall effect: a hierarchy of incompressible quantum fluid states," Physical Review Letters, vol. 51, no. 7, pp. 605-608, 1983.

[6] B. I. Halperin, "Statistics of quasiparticles and the hierarchy of fractional quantized Hall states," Physical Review Letters, vol. 52, no. 18, pp. 1583-1586, 1984.

[7] S. M. Girvin, "Particle-hole symmetry in the anomalous quantum Hall effect," Physical Review B, vol. 29, no. 10, pp. 6012-6014, 1984.

[8] J. K. Jain, "Composite-fermion approach for the fractional quantum Hall effect," Physical Review Letters, vol. 63, no. 2, pp. 199-202, 1989.

[9] J. K. Jain, "Theory of the fractional quantum Hall effect," Physical Review B, vol. 41, no. 11, pp. 7653-7665, 1990.

[10] A. Wójs and J. J. Quinn, "Quasiparticle interactions in fractional quantum Hall systems: justification of different hierarchy schemes," Physical Review B, vol. 61, no. 4, pp. 2846-2854, 2000.

[11] A. Wójs, K.-S. Yi, and J. J. Quinn, "Fractional quantum Hall states of clustered composite fermions," Physical Review B, vol. 69, Article ID 205322, 13 pages, 2004.

[12] J. H. Smet, "Wheels within wheels," Nature, vol. 422, no. 6930, pp. 391-392, 2003.

[13] M. R. Peterson and J. K. Jain, "Flavor altering excitations of composite fermions," Physical Review Letters, vol. 93, no. 4, Article ID 046402, 4 pages, 2004.

[14] E. A. Pashitskii, "New quantum states in the fractional quantum Hall effect regime," Low Temperature Physics, vol. 31, no. 2, pp. 171-178, 2005.

[15] R. Tao and D. J. Thouless, "Fractional quantization of Hall conductance," Physical Review B, vol. 28, no. 2, pp. 1142-1144, 1983.

[16] R. Tao, "Fractional quantization of Hall conductance. II," Physical Review B, vol. 29, no. 2, pp. 636-644, 1984.

[17] S. Sasaki, "Gap structure and gapless structure in fractional quantum Hall effect," Advances in Condensed Matter Physics, vol. 2012, Article ID 281371, 13 pages, 2012.

[18] S. Sasaki, "Energy gap in fractional quantum Hall effect," Physica B, vol. 281-282, pp. 838-839, 2000.

[19] S. Sasaki, "Binding energy and polarization of fractional quantum Hall state," in Proceedings of the 25th International Conference on the Physics of Semiconductors, pp. 925-926, Springer, 2011.

[20] S. Sasaki, "Spin polarization in fractional quantum Hall effect," Surface Science, vol. 532-535, pp. 567-575, 2003.
[21] S. Sasaki, "Calculation of binding energies for fractional quantum Hall states with even denominators," http://arxiv.org/abs/ cond-mat/0703360.

[22] S. Sasaki, "Energy gaps in fractional quantum Hall states," Journal of Physics, vol. 100, no. 4, Article ID 042021, 4 pages, 2008.

[23] S. Sasaki, "Energy spectra for fractional quantum Hall states," http://arxiv.org/abs/0708.1541.

[24] W. Pan, R. R. Du, H. L. Stormer et al., "Strongly anisotropic electronic transport at Landau level filling factor $v=9 / 2$ and $\nu=5 / 2$ under a tilted magnetic field," Physical Review Letters, vol. 83, no. 4, pp. 820-823, 1999.

[25] M. P. Lilly, K. B. Cooper, J. P. Eisenstein, L. N. Pfeiffer, and K. W. West, "Evidence for an anisotropic state of two-dimensional electrons in high Landau levels," Physical Review Letters, vol. 82, pp. 394-397, 1999.

[26] J. P. Eisenstein, K. B. Cooper, L. N. Pfeiffer, and K. W. West, "Insulating and fractional quantum Hall states in the first excited Landau level," Physical Review Letters, vol. 88, no. 7, Article ID 076801, 4 pages, 2002.

[27] C. R. Dean, B. A. Piot, P. Hayden et al., "Intrinsic gap of the $v=$ 5/2 fractional quantum hall state," Physical Review Letters, vol. 100, no. 14, Article ID 146803, 2008.

[28] J. S. Xia, W. Pan, C. L. Vicente et al., "Electron correlation in the second Landau level: a competition between many nearly degenerate quantum phases," Physical Review Letters, vol. 93, no. 17, Article ID 176809, 4 pages, 2004.

[29] G. A. Csáthy, J. S. Xia, C. L. Vicente et al., "Tilt-induced localization and delocalization in the second Landau level," Physical Review Letters, vol. 94, no. 14, Article ID 146801, 4 pages, 2005.

[30] W. Pan, J. S. Xia, H. L. Stormer et al., "Experimental studies of the fractional quantum Hall effect in the first excited Landau level," Physical Review B, vol. 77, no. 7, Article ID 075307, 2008.

[31] H. C. Choi, W. Kang, S. Das Sarma, L. N. Pfeiffer, and K. W. West, "Activation gaps of fractional quantum Hall effect in the second Landau level," Physical Review B, vol. 77, no. 8, Article ID 081301, 4 pages, 2008.

[32] I. L. Aleiner and L. I. Glazman, "Two-dimensional electron liquid in a weak magnetic field," Physical Review B, vol. 52, no. 15, pp. 11296-11312, 1995.

[33] A. A. Koulakov, M. M. Fogler, and B. I. Shklovskii, "Charge density wave in two-dimensional electron liquid in weak magnetic field," Physical Review Letters, vol. 76, no. 3, pp. 499-502, 1996.

[34] R. Moessner and J. T. Chalker, "Exact results for interacting electrons in high Landau levels," Physical Review B, vol. 54, no. 7, pp. 5006-5015, 1996.

[35] E. H. Rezayi, F. D. M. Haldane, and K. Yang, "Charge-densitywave ordering in half-filled high Landau levels," Physical Review Letters, vol. 83, no. 6, pp. 1219-1222, 1999.

[36] F. D. M. Haldane and E. H. Rezayi, "Spin-singlet wave function for the half-integral quantum Hall effect," Physical Review Letters, vol. 60, no. 10, pp. 956-959, 1988.

[37] G. Moore and N. Read, "Nonabelions in the fractional quantum Hall effect," Nuclear Physics B, vol. 360, no. 2-3, pp. 362-396, 1991.

[38] N. Read, "Paired fractional quantum Hall states and the $v=5 / 2$ puzzle," Physica B, vol. 298, no. 1-4, pp. 121-128, 2001.

[39] M. Greiter, X. G. Wen, and F. Wilczek, "Paired Hall state at half filling," Physical Review Letters, vol. 66, no. 24, pp. 3205-3208, 1991. 
[40] M. Greiter, X. G. Wen, and F. Wilczek, "Paired Hall states," Nuclear Physics B, vol. 374, no. 3, pp. 567-614, 1992.

[41] R. H. Morf, "Transition from quantum Hall to compressible states in the second Landau level: new light on the $v=5 / 2$ Enigma," Physical Review Letters, vol. 80, no. 7, pp. 1505-1508, 1998.

[42] E. H. Rezayi and F. D. M. Haldane, "Incompressible paired Hall state, stripe order, and the composite fermion liquid phase in half-filled Landau levels," Physical Review Letters, vol. 84, no. 20, pp. 4685-4688, 2000.

[43] A. C. Balram, Y. H. Wu, G. J. Sreejith, A. Wójs, and J. K. Jain, "Role of exciton screening in the 7/3 fractional quantum Hall effect," Physical Review Letters, vol. 110, no. 18, Article ID 186801, 5 pages, 2013.

[44] W. Pan, H. L. Stormer, D. C. Tsui, L. N. Pfeiffer, K. W. Baldwin, and K. W. West, "Experimental evidence for a spin-polarized ground state in the $\nu=5 / 2$ fractional quantum Hall effect," Solid State Communications, vol. 119, no. 12, pp. 641-645, 2001.

[45] C. Zhang, C. Huan, J. S. Xia et al., "Spin polarization of the $v=$ 12/5 fractional quantum Hall state," Physical Review B, vol. 85, no. 24, Article ID 241302, 5 pages, 2012.

[46] W. Pan, K. W. Baldwin, K. W. West, L. N. Pfeiffer, and D. C. Tsui, "Spin Transition in the $v=8 / 3$ fractional quantum Hall effect," Physical Review Letters, vol. 108, no. 21, Article ID 216804, 5 pages, 2012.

[47] A. E. Feiguin, E. Rezayi, K. Yang, C. Nayak, and S. Das Sarma, "Spin polarization of the $v=5 / 2$ quantum Hall state," Physical Review B, vol. 79, no. 11, Article ID 115322, 2009.

[48] W. Zhu, D. N. Sheng, and F. D. M. Haldane, "Minimal entangled states and modular matrix for fractional quantum Hall effect in topological flat bands," Physical Review B, vol. 88, no. 3, Article ID 035122, 6 pages, 2013.

[49] J. Biddle, R. M. Peterson, and S. Das Sarma, "Variational Monte Carlo study of spin-polarization stability of fractional quantum Hall states against realistic effects in half-filled Landau levels," Physical Review B, vol. 87, no. 23, Article ID 235134, 8 pages, 2013.

[50] I. V. Kukushkin, K. V. Klitzing, and K. Eberl, "Spin polarization of composite fermions: measurements of the Fermi energy," Physical Review Letters, vol. 82, no. 18, pp. 3665-3668, 1999.

[51] S. Sasaki, "Solvable models of spin-1/2 chains with an energy gap," Physical Review E, vol. 53, no. 1, pp. 168-178, 1996.

[52] R. E. Peierls, Quantum Theory of Solids, Oxford University, London, UK, 1955.

[53] S. Sasaki, "Spin-Peierls effect in spin-polarization of fractional quantum Hall states," Surface Science, vol. 566-568, no. 1-3, pp. 1040-1046, 2004.

[54] S. Sasaki, Surface Science: New Research, chapter 4, Nova Science, 2006. 

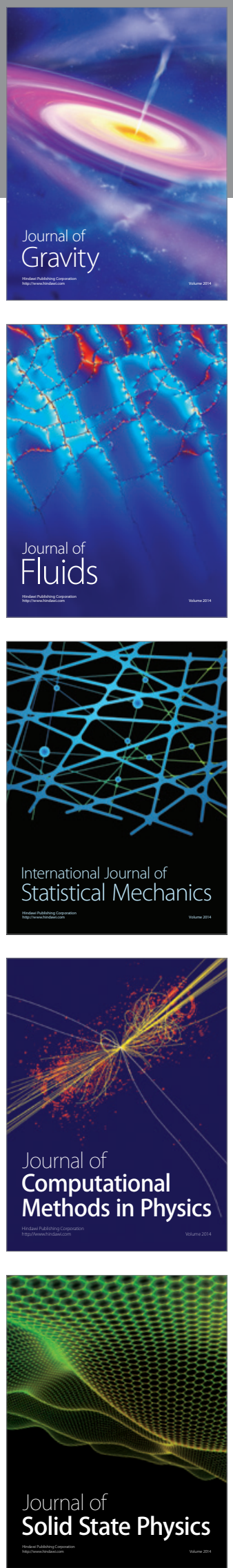

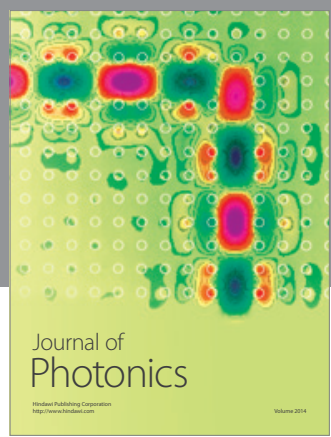

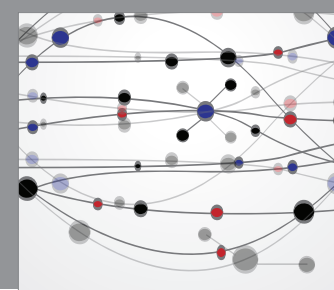

The Scientific World Journal

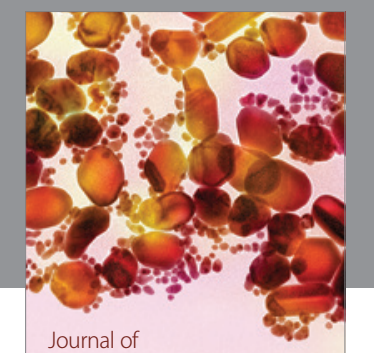

Soft Matter
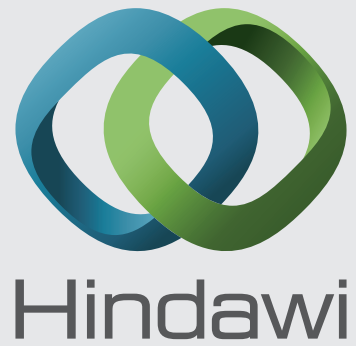

Submit your manuscripts at

http://www.hindawi.com
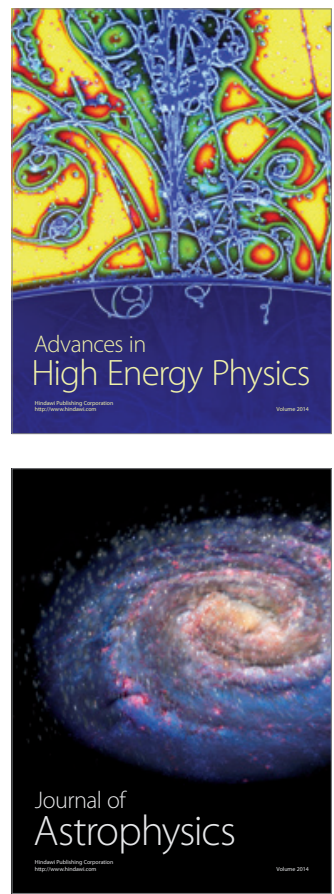
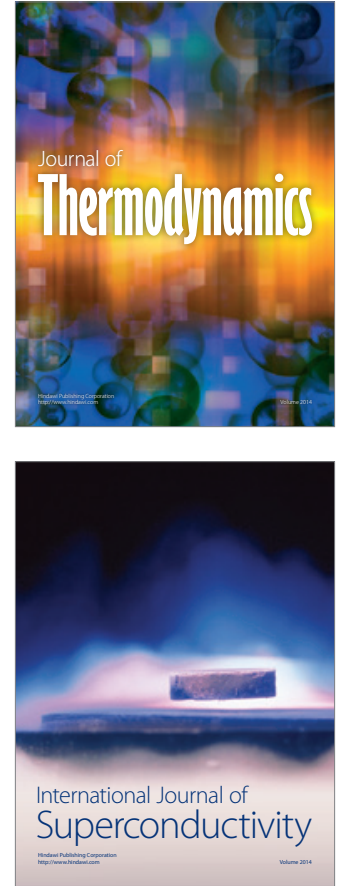
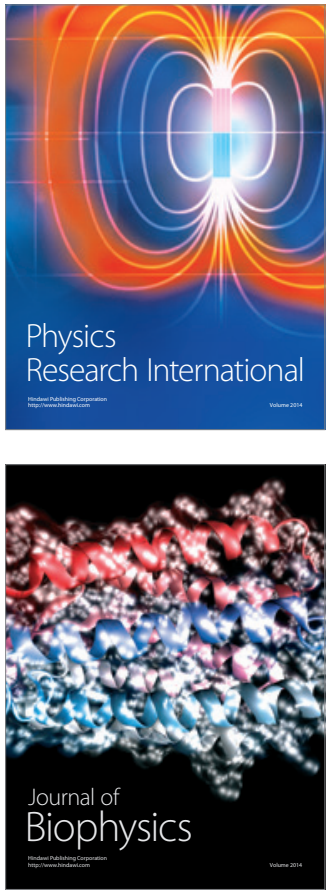
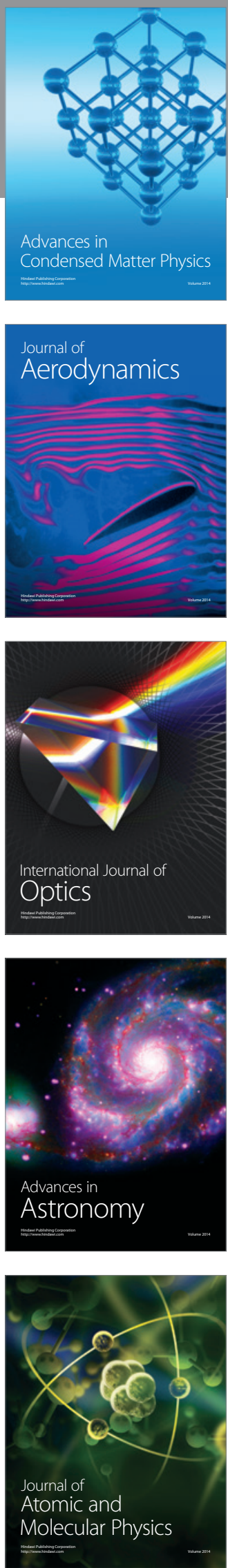